\title{
Hybrid static potential flux tubes from SU(2) and SU(3) lattice gauge theory
}

\author{
Lasse Müller®, Owe Philipsen๑, Christian Reisinger, and Marc Wagner® \\ Goethe-Universität Frankfurt am Main, Institut für Theoretische Physik, Max-von-Laue-Straße 1, \\ D-60438 Frankfurt am Main, Germany
}

(Received 15 July 2019; published 16 September 2019)

\begin{abstract}
We compute chromoelectric and chromomagnetic flux densities for hybrid static potentials in $\mathrm{SU}(2)$ and SU(3) lattice gauge theory. In addition to the ordinary static potential with quantum numbers $\Lambda_{\eta}^{\epsilon}=\Sigma_{g}^{+}$, we present numerical results for seven hybrid static potentials corresponding to $\Lambda_{\eta}^{(e)}=\Sigma_{u}^{+}, \Sigma_{g}^{-}$, $\Sigma_{u}^{-}, \Pi_{g}, \Pi_{u}, \Delta_{g}, \Delta_{u}$, where the flux densities of five of them are studied for the first time in this work. We observe hybrid static potential flux tubes, which are significantly different from that of the ordinary static potential. They are reminiscent of vibrating strings, with localized peaks in the flux densities that can be interpreted as valence gluons.
\end{abstract}

DOI: $10.1103 /$ PhysRevD.100.054503

\section{INTRODUCTION}

The majority of mesons, i.e., hadrons with integer total angular momentum, are quark-antiquark pairs. It is, however, expected that some mesons, so-called exotic mesons, have a more complicated composition in terms of quarks and gluons. An important example is hybrid mesons, where gluons contribute to the quantum numbers $J^{P C}$ ( $J$ : total angular momentum; $P$ : parity; $C$ : charge conjugation) in a nontrivial way. In the quark model, where mesons are quark-antiquark pairs, quantum numbers are restricted to $P=(-1)^{L+1}$ and $C=(-1)^{L+S}$ with spin $S=0,1$ and orbital angular momentum $L=0,1,2, \ldots$. Thus, mesons with $J^{P C}=0^{+-}, 0^{--}, 1^{-+}, 2^{+-}, \ldots$, which are not allowed in the quark model, are obvious candidates for exotic mesons like hybrids. Moreover, a higher density of states than obtained by the quark model might also indicate hybrid mesons.

Experimentally observed examples, which could be hybrid mesons, are the $J^{P C}=1^{-+}$states $\pi_{1}(1400)$ and $\pi_{1}(1600)$. They could, however, also be tetraquarks, i.e., two quarks and two antiquarks without excited glue. For heavy-heavy mesons, the situation seems to be even less clear. There are several exotic candidates, which could be hybrid mesons, but for none of them does such an interpretation seem to be likely (see, e.g., the experimental review of exotic hadrons [1] and the discussion in Sec. VII.A of Ref. [2]). Thus, the search for gluonic excitations is an important part of the research program

Published by the American Physical Society under the terms of the Creative Commons Attribution 4.0 International license. Further distribution of this work must maintain attribution to the author(s) and the published article's title, journal citation, and DOI. Funded by SCOAP ${ }^{3}$. of current and future experiments, e.g., the GlueX experiment at the JLab accelerator or the PANDA experiment at the FAIR accelerator.

Also on the theoretical side, there are many open questions concerning hybrid mesons (see, e.g., the theoretical reviews [3-6]). They are difficult to study because in QCD total angular momentum $J$ and parity $P$ are not separately conserved for gluons on the one hand and for the quark-antiquark pair on the other hand. Only the overall $J^{P}$ are quantum numbers. For heavy hybrid mesons, e.g., composed of a $b$ and a $\bar{b}$ quark and gluons, a simplification and good approximation is to study the static limit. In that limit, the quark positions are frozen, which allows one to separate the treatment of gluons and quarks.

In this work, we use $\mathrm{SU}(2)$ and $\mathrm{SU}(3)$ lattice gauge theory to study heavy hybrid mesons in the static limit. For quite some time, hybrid static potentials haven been computed by various groups, mainly with the intention to compute masses of heavy hybrid mesons using the BornOppenheimer approximation (see Refs. [7-31] and the recent review article [32]). We focus on a different problem, the computation of the gluonic flux densities for hybrid potential states, i.e., the structure of the flux tube, for several hybrid channels. While such flux tubes have been studied for the ordinary static potential using lattice gauge theory for quite some time (see Refs. [33-48]), this is a rather new direction for hybrid static potentials, where first results appeared only recently [49-52]. In this paper, we substantially extend existing work by performing computations for seven hybrid static potential sectors characterized by quantum numbers $\Lambda_{\eta}^{(e)}=\Sigma_{u}^{+}, \Sigma_{g}^{-}, \Sigma_{u}^{-}, \Pi_{g}$, $\Pi_{u}, \Delta_{g}, \Delta_{u}$. Five of these sectors are studied for the first time, where preliminary results have been presented at a recent conference [52]. 
The paper is structured as follows. In Sec. II, we discuss theoretical basics, including quantum numbers for hybrid static potentials, the construction of corresponding trial states, and the computation of chromoelectric and chromomagnetic flux densities. Section III contains a brief summary of our lattice setup. In Sec. IV, we present our numerical results. We start with a discussion of systematic errors and symmetries, before showing and interpreting our main results, the chromoelectric and chromomagnetic flux densities for the seven hybrid static potential sectors $\Lambda_{\eta}^{(\epsilon)}=\Sigma_{u}^{+}, \Sigma_{g}^{-}, \Sigma_{u}^{-}, \Pi_{g}, \Pi_{u}, \Delta_{g}, \Delta_{u}$. In Sec. V, we conclude with a short summary and an outlook.

\section{HYBRID STATIC POTENTIALS AND FLUX TUBES}

\section{A. Hybrid static potential quantum numbers and trial states}

A hybrid static potential is the potential of a static quark $Q$ and a static antiquark $\bar{Q}$, where the gluons form nontrivial structures and thus contribute to the quantum numbers. Such potentials can be computed from temporal correlation functions of hybrid static potential trial states. After replacing the static quark operators by corresponding propagators, these correlation functions are similar to Wilson loops. Instead of straight spatial Wilson lines, there are, however, parallel transporters with more complicated spatial structures. For a detailed discussion of such correlation functions, see, e.g., our recent work [31], where we have carried out a precision computation of hybrid static potentials using $\mathrm{SU}(3)$ lattice gauge theory.

In the following, we consider a static quark and a static antiquark located at positions $\mathbf{r}_{Q}=(0,0,+r / 2)$ and $\mathbf{r}_{\bar{Q}}=(0,0,-r / 2)$, respectively; i.e., they are separated along the $z$ axis. We omit the $x$ and the $y$ coordinates, i.e., $Q(+r / 2) \equiv Q(0,0,+r / 2)$ and $\bar{Q}(-r / 2) \equiv \bar{Q}(0,0,-r / 2)$.

Hybrid static potentials can be characterized by the following quantum numbers:

(i) $\Lambda=0,1,2, \ldots$, the absolute value of the total angular momentum with respect to the $Q \bar{Q}$ separation axis, i.e., with respect to the $z$ axis.

(ii) $\eta=+,-$, the eigenvalue corresponding to the operator $\mathcal{P} \circ \mathcal{C}$, i.e., the combination of parity and charge conjugation.

(iii) $\epsilon=+,-$, the eigenvalue corresponding to the operator $\mathcal{P}_{x}$, which denotes the spatial reflection along the $x$ axis (an axis perpendicular to the $Q \bar{Q}$ separation axis).

It is common convention to write $\Lambda=\Sigma, \Pi, \Delta$ instead of $\Lambda=0,1,2$ and $\eta=g, u$ ("gerade," "ungerade") instead of $\eta=+,-$. Note that for absolute total angular momentum $\Lambda \geq 1$ the spectrum is degenerate with respect to $\epsilon=+$ and $\epsilon=-$; i.e., there are pairs of identical hybrid static potentials. Thus, the labeling of hybrid static potentials is typically $\Lambda_{\eta}^{\epsilon}$ for $\Lambda=0=\Sigma$ and $\Lambda_{\eta}$ for $\Lambda \geq 1$.
In Ref. [31], we discussed hybrid static potential creation operators and trial states both in the continuum and in lattice gauge theory in detail and performed a comprehensive optimization of these operators in $\mathrm{SU}(3)$ lattice gauge theory. In this paper, we use the information obtained during this optimization to define suitable hybrid static potential creation operators both for $\mathrm{SU}(2)$ and $\mathrm{SU}(3)$ lattice gauge theory. These operators are important building blocks of the two-point and three-point functions, which need to be computed for the investigation of hybrid static potential flux tubes (see Sec. II B).

Our trial states, which have definite quantum numbers $\Lambda_{\eta}^{\epsilon}$, are

$\left|\Psi_{\Lambda_{\eta}^{e}}(r)\right\rangle=\bar{Q}(-r / 2) a_{S ; \Lambda_{\eta}^{\epsilon}}(-r / 2,+r / 2) Q(+r / 2)|\Omega\rangle$

with creation operators

$$
\begin{aligned}
& a_{S ; \Lambda_{\eta}^{e}}(-r / 2,+r / 2) \\
& =\frac{1}{4} \sum_{k=0}^{3} \exp \left(\frac{i \pi \Lambda k}{2}\right) R\left(\frac{\pi k}{2}\right)\left(U ( - r / 2 , r _ { 1 } ) \left(S\left(r_{1}, r_{2}\right)\right.\right. \\
& \left.\quad+\epsilon S_{\mathcal{P}_{x}}\left(r_{1}, r_{2}\right)\right) U\left(r_{2},+r / 2\right) \\
& \quad+U\left(-r / 2,-r_{2}\right)\left(\eta S_{\mathcal{P} \circ \mathcal{C}}\left(-r_{2},-r_{1}\right)\right. \\
& \left.\left.\quad+\eta \epsilon S_{\left(\mathcal{P}_{\circ}\right) \mathcal{P}_{x}}\left(-r_{2},-r_{1}\right)\right) U\left(-r_{1},+r / 2\right)\right) .
\end{aligned}
$$

$U\left(-r / 2, r_{1}\right) S\left(r_{1}, r_{2}\right) U\left(r_{2},+r / 2\right)$ is a product of link variables connecting the quark and the antiquark in a gauge invariant way, where both $U\left(-r / 2, r_{1}\right)$ and $U\left(r_{2},+r / 2\right)$ are straight lines on the $z$ axis, while $S\left(r_{1}, r_{2}\right)$ has a more complicated shape. $S_{\mathcal{P} \circ \mathcal{C}}\left(-r_{2},-r_{1}\right)$ is the spatial reflection of $S\left(r_{1}, r_{2}\right)$ combined with charge conjugation, $S_{\mathcal{P}_{x}}\left(r_{1}, r_{2}\right)$ is the spatial reflection of $S\left(r_{1}, r_{2}\right)$ along the $x$ axis, and $S_{(\mathcal{P} \circ \mathcal{C}) \mathcal{P}_{x}}\left(-r_{2},-r_{1}\right)$ is the combination of both operations. $R(\varphi)$ represents the rotation operator with respect to the $Q \bar{Q}$ separation axis with angle $\varphi$, which acts on the shapes $S$, and $\sum_{k=0}^{3}$ denotes the sum over the four angles allowed on a cubic lattice, i.e., $\varphi=0, \pi / 2, \pi, 3 \pi / 2$. Thus, $\sum_{k=0}^{3} \exp (i \pi \Lambda k / 2) R(\pi k / 2)$ is the lattice analogue of $\int d \varphi \exp (i \Lambda \varphi) R(\varphi)$ in the continuum. While the latter generates a definite $z$ component of angular momentum, the former is a projection to one of the irreducible representations of cubic rotations around a fixed axis, where each receives contributions from an infinite number of angular momentum sectors. For details, see Sec. 2 of Ref. [31].

$U\left(-r / 2, r_{1}\right) S\left(r_{1}, r_{2}\right) U\left(r_{2},+r / 2\right)$ has been optimized in Ref. [31], such that the overlap of $\left|\Psi_{\Lambda_{\eta}^{e}}(r)\right\rangle$ to the ground state in the $\Lambda_{\eta}^{\epsilon}$ sector is rather large. In contrast to Ref. [31], we use in this work only a single operator $S$ for each $\Lambda_{\eta}^{\epsilon}$ sector, not a linear combination obtained by a variational analysis. This reduces computation time to a feasible level, while the suppression of excited states in the two-point 
TABLE I. Optimized creation operators for $\Lambda_{\eta}^{\epsilon}=\Sigma_{u}^{+}, \Sigma_{g}^{-}, \Sigma_{u}^{-}, \Pi_{g}^{-}, \Pi_{u}^{+}, \Delta_{g}^{ \pm}, \Delta_{u}^{ \pm}$. The equation in the caption of each table defines a creation operator. The left-hand side represents $U\left(-r / 2, r_{1}\right) S\left(r_{1}, r_{2}\right) U\left(r_{2},+r / 2\right)$ from Eq. (2), where lower indices are chosen such that the notation is consistent with our previous work [31]. The right-hand side is a product of connected gauge links, where $U_{j}^{E}$ denotes the multiplication of $E$ neighboring gauge links in the $j$ direction. Note that, even though the $\Pi_{\eta}$ and the $\Delta_{\eta}$ hybrid potentials are degenerate with respect to $\epsilon$, the construction of creation operators via Eq. (2) is not independent of $\epsilon$. One can obtain an optimized $\Pi_{\eta}^{-}$operator from an optimized $\Pi_{\eta}^{+}$operator by applying a $\pi / 2$ rotation with respect to the $z$ axis. For $\Delta_{\eta}^{\epsilon}$ operators, there is no analogous simple prescription. Therefore, we provide four different optimized $\Delta_{\eta}^{\epsilon}$ operators.

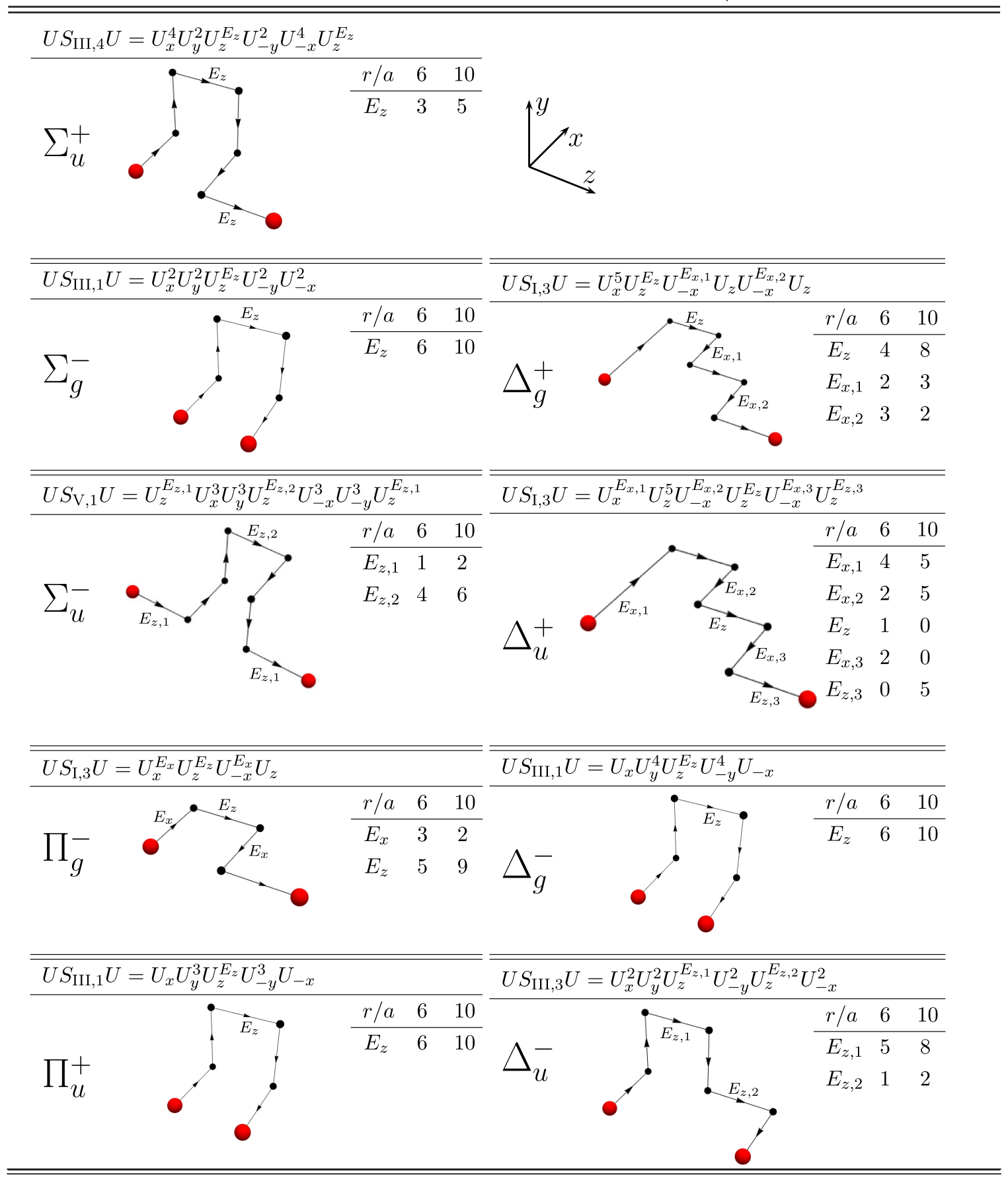

and three-point functions is still sufficiently strong. $U\left(-r / 2, r_{1}\right) S\left(r_{1}, r_{2}\right) U\left(r_{2},+r / 2\right)$ is different for each of the eight $\Lambda_{\eta}^{\epsilon}$ sectors as well as for the two $Q \bar{Q}$ separations $r=6 a, 10 a$ considered. For the $\Sigma_{g}^{+}$sector, i.e., the ordinary static potential, it is just a straight line, while for $\Lambda_{\eta}^{\epsilon}=\Sigma_{u}^{+}, \Sigma_{g}^{-}, \Sigma_{u}^{-}, \Pi_{g}^{-}, \Pi_{u}^{+}, \Delta_{g}^{ \pm}, \Delta_{u}^{ \pm}$, details are collected in Table I. For each $\Lambda_{\eta}^{\epsilon}$ sector, we take that operator from the set of three to four operators we optimized in Ref. [31], 
which minimizes the effective potential at $t=a$. Thus, Table I contains that part of the information shown in Tables 1 to 7 of Ref. [31], which is relevant in the context of this work.

\section{B. Expectation values of squared field strength components}

The energy density of the gluon field is

$$
\begin{aligned}
\mathcal{E}(\mathbf{x})= & \frac{1}{8 \pi}\left(\sum_{j=x, y, z} \sum_{a} E_{j}^{a}(\mathbf{x}) E_{j}^{a}(\mathbf{x})\right. \\
& \left.+\sum_{j=x, y, z} \sum_{a} B_{j}^{a}(\mathbf{x}) B_{j}^{a}(\mathbf{x})\right),
\end{aligned}
$$

where $E_{j}^{a}(\mathbf{x})$ and $B_{j}^{a}(\mathbf{x})$ denote the components of the chromoelectric and chromomagnetic field strengths with spatial indices $j$ and color indices $a[a=1, \ldots, 3$ for gauge group $\mathrm{SU}(2)$ and $a=1, \ldots, 8$ for gauge group $\mathrm{SU}(3)]$. The main goal of this work is to compute the expectation values of the six gauge invariant terms $F_{j}^{2}(\mathbf{x})=\sum_{a} F_{j}^{a}(\mathbf{x}) F_{j}^{a}(\mathbf{x})$ (no sum over $j ; F_{j}^{a}=E_{j}^{a}$ or $F_{j}^{a}=B_{j}^{a}$ ) contributing to
Eq. (3) for states with a static quark-antiquark pair and quantum numbers $\Lambda_{\eta}^{\epsilon}$. These chromoelectric and chromomagnetic flux densities provide information about the shapes of hybrid static potential flux tubes and the gluonic energy distributions inside heavy-heavy hybrid mesons.

To compute the flux densities, we need the following quantities:

(i) Two-point correlation functions:

$$
\begin{aligned}
W_{\Lambda_{\eta}^{e}}\left(r, t_{2}, t_{0}\right)= & \left\langle\Psi_{\Lambda_{\eta}^{\varepsilon}}\left(r, t_{2}\right) \mid \Psi_{\Lambda_{\eta}^{e}}\left(r, t_{0}\right)\right\rangle \\
= & \sum_{m}\left|\left\langle\Psi_{\Lambda_{\eta}^{e}}(r) \mid m_{\Lambda_{\eta}^{e}}(r)\right\rangle\right|^{2} \\
& \times e^{-\left(E_{m, \Lambda_{\eta}^{\epsilon}}(r)-E_{\Omega}\right)\left(t_{2}-t_{0}\right)},
\end{aligned}
$$

where $t_{2}>t_{0}$ and $\left|m_{\Lambda_{\eta}^{\epsilon}}(r)\right\rangle$ denotes the $m$ th energy eigenstate with a static quark $Q$ and a static antiquark $\bar{Q}$ at positions $(0,0,+r / 2)$ and $(0,0,-r / 2)$ and quantum numbers $\Lambda_{\eta}^{\epsilon} . E_{m, \Lambda_{\eta}^{\epsilon}}(r)$ is the corresponding energy eigenvalue, where $E_{0, \Lambda_{\eta}^{e}}(r)<E_{1, \Lambda_{\eta}^{e}}(r)<E_{2, \Lambda_{\eta}^{e}}(r)<\ldots$ The static potential with quantum numbers $\Lambda_{\eta}^{\epsilon}$ is defined as $V_{\Lambda_{\eta}^{\epsilon}}(r)=E_{0, \Lambda_{\eta}^{\epsilon}}(r)-E_{\Omega}$.

(ii) Three-point correlation functions:

$$
\begin{aligned}
C_{\Lambda_{\eta}^{e}, F_{j}^{2}}\left(r, t_{2}, t_{0} ; \mathbf{x}, t_{1}\right)= & \left\langle\Psi_{\Lambda_{\eta}^{\epsilon}}\left(r, t_{2}\right)\left|F_{j}^{2}\left(\mathbf{x}, t_{1}\right)\right| \Psi_{\Lambda_{\eta}^{\epsilon}}\left(r, t_{0}\right)\right\rangle \\
= & \sum_{m, n}\left\langle\Psi_{\Lambda_{\eta}^{\epsilon}}(r) \mid m_{\Lambda_{\eta}^{\epsilon}}(r)\right\rangle\left\langle m_{\Lambda_{\eta}^{\epsilon}}(r)\left|F_{j}^{2}(\mathbf{x})\right| n_{\Lambda_{\eta}^{\epsilon}}(r)\right\rangle\left\langle n_{\Lambda_{\eta}^{e}}(r) \mid \Psi_{\Lambda_{\eta}^{\epsilon}}(r)\right\rangle \\
& \times e^{-\left(E_{m, \Lambda_{\eta}^{\epsilon}}(r)-E_{\Omega}\right)\left(t_{2}-t_{1}\right)} e^{-\left(E_{n, \Lambda_{\eta}^{\epsilon}}(r)-E_{\Omega}\right)\left(t_{1}-t_{0}\right)},
\end{aligned}
$$

where $t_{2}>t_{1}>t_{0}$.

(iii) Vacuum expectation values:

$$
\left\langle\Omega\left|F_{j}^{2}\right| \Omega\right\rangle .
$$

These quantities can be combined with expressions for the expectation values of $E_{j}^{2}(\mathbf{x})$ and $B_{j}^{2}(\mathbf{x})$ for static potential states with quantum numbers $\Lambda_{\eta}^{\epsilon}$ with the vacuum expectation value subtracted:

$$
\begin{aligned}
\Delta F_{j, \Lambda_{\eta}^{\epsilon}}^{2}(r ; \mathbf{x}) & =\left\langle 0_{\Lambda_{\eta}^{\epsilon}}(r)\left|F_{j}^{2}(\mathbf{x})\right| 0_{\Lambda_{\eta}^{\epsilon}}(r)\right\rangle-\left\langle\Omega\left|F_{j}^{2}\right| \Omega\right\rangle \\
& =\lim _{t_{2}-t_{1}, t_{1}-t_{0} \rightarrow \infty} \underbrace{\frac{C_{\Lambda_{\eta}^{\epsilon}, F_{j}^{2}}\left(r, t_{2}, t_{0} ; \mathbf{x}, t_{1}\right)}{W_{\Lambda_{\eta}^{\epsilon}}\left(r, t_{2}, t_{0}\right)}-\left\langle\Omega\left|F_{j}^{2}\right| \Omega\right\rangle}_{=\Delta F_{\text {eff } j ;, \Lambda_{\eta}^{\epsilon}}^{2}\left(r, t_{2}, t_{0} ; \mathbf{x}, t_{1}\right)}
\end{aligned}
$$

(see also Ref. [33], where this quantity was first defined and used to study flux densities for the ordinary static potential with quantum numbers $\Lambda_{\eta}^{\epsilon}=\Sigma_{g}^{+}$).

The right-hand side of Eq. (7) can be evaluated using Euclidean lattice gauge theory path integrals,

$$
\Delta E_{\mathrm{eff} ; j, \Lambda_{\eta}^{e}}^{2}\left(r, t_{2}, t_{0} ; \mathbf{x}, t_{1}\right)=+\left(\frac{\left\langle\tilde{W}\left(r, t_{2}, t_{0}\right) \cdot P_{0 j}\left(\mathbf{x}, t_{1}\right)\right\rangle_{U}}{\left\langle\tilde{W}\left(r, t_{2}, t_{0}\right)\right\rangle_{U}}-\left\langle P_{0 j}\right\rangle_{U}\right)
$$




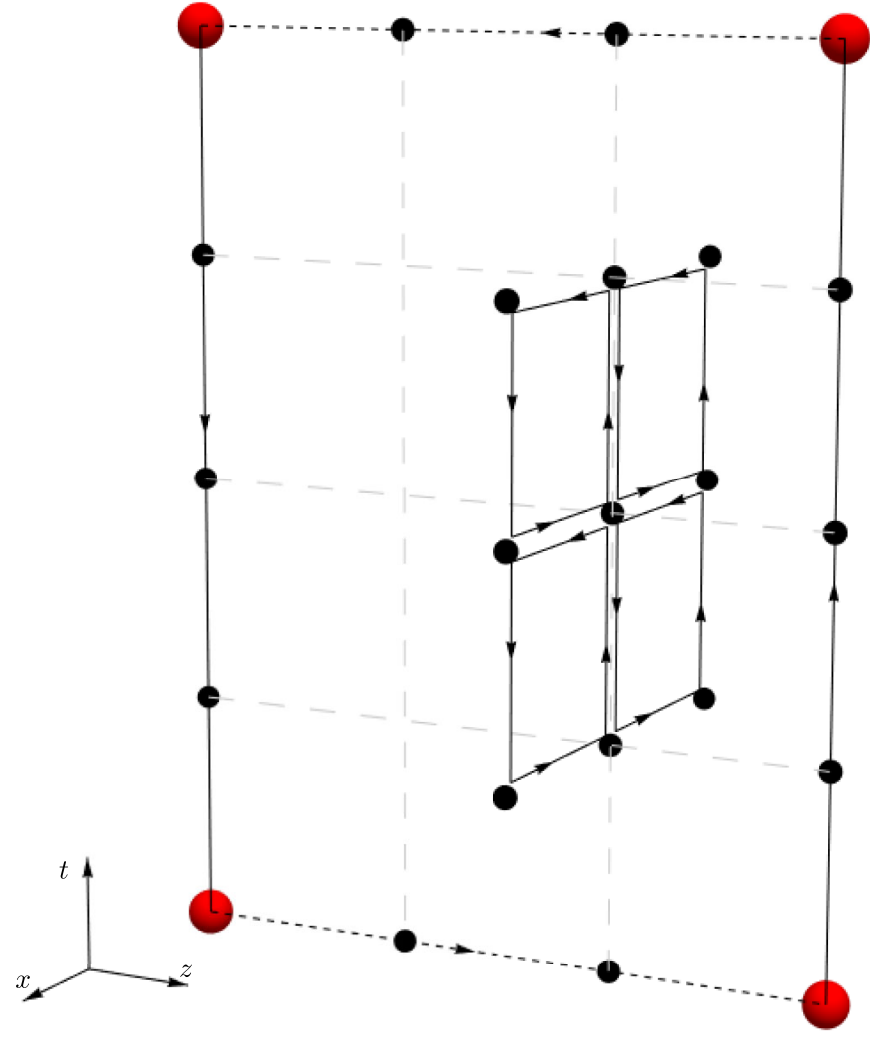

(a)

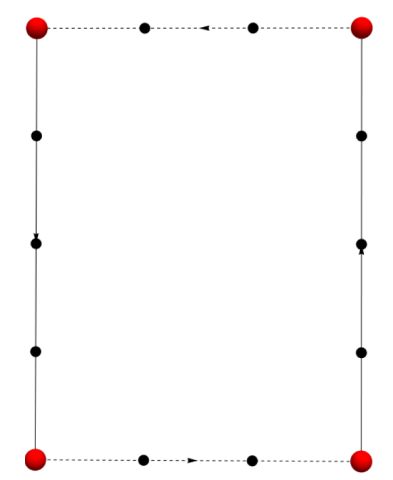

(b)

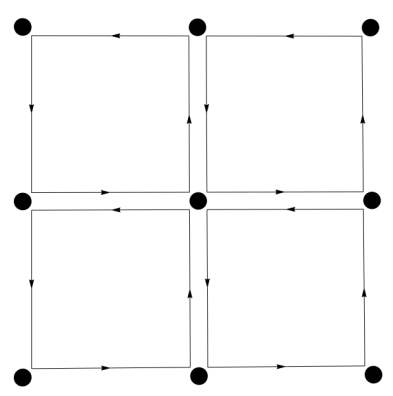

(c)

FIG. 1. Graphical illustration of lattice field theory quantities needed to determine $\Delta E_{j, \Lambda_{\eta}^{\epsilon}}^{2}(r ; \mathbf{x})$ and $\Delta B_{j, \Lambda_{\eta}^{\epsilon}}^{2}(r ; \mathbf{x})$. Red spheres, black dots, black solid lines, and black dashed lines represent static (anti)quarks, lattice sites, gauge links, and operators $a_{S ; \Lambda_{\eta}^{e}}$, respectively. (a) $\tilde{W}\left(r, t_{2}, t_{0}\right) \cdot P_{0 x}\left(\mathbf{x}, t_{1}\right)$ for $r=3 a, t_{2}-t_{0}=4 a, t_{1}=\left(t_{2}-t_{0}\right) / 2$ (gray dashed lines parallel to the $t$ axis and the $z$ axis are drawn to guide the eye). (b) The corresponding Wilson loop $\tilde{W}\left(r, t_{2}, t_{0}\right)$. (c) The symmetrized plaquette $P_{\mu \nu}$.

$$
\begin{aligned}
\Delta & B_{\mathrm{eff} ; j, \Lambda_{\eta}^{\epsilon}}^{2}\left(r, t_{2}, t_{0} ; \mathbf{x}, t_{1}\right) \\
& =-\left(\frac{\left\langle\tilde{W}\left(r, t_{2}, t_{0}\right) \cdot\left|\epsilon_{j k l} / 2\right| P_{k l}\left(\mathbf{x}, t_{1}\right)\right\rangle_{U}}{\left\langle\tilde{W}\left(r, t_{2}, t_{0}\right)\right\rangle_{U}}-\left\langle\left|\epsilon_{j k l} / 2\right| P_{k l}\right\rangle_{U}\right),
\end{aligned}
$$

where $\langle\ldots\rangle_{U}$ denotes the path integral expectation value and

$$
\begin{aligned}
\tilde{W}\left(r, t_{2}, t_{0}\right)= & \operatorname{Tr}\left(a_{S ; \Lambda_{\eta}^{e}}\left(-r / 2,+r / 2 ; t_{0}\right) U\left(+r / 2 ; t_{0}, t_{2}\right)\right. \\
& \left.\times\left(a_{S ; \Lambda_{\eta}^{e}}\left(-r / 2,+r / 2 ; t_{2}\right)\right)^{\dagger} U\left(-r / 2 ; t_{2}, t_{0}\right)\right)
\end{aligned}
$$

(for $\Lambda_{\eta}^{\epsilon}=\Sigma_{g}^{+}$, i.e., the ordinary static potential, $\tilde{W}\left(r, t_{2}, t_{0}\right)$ is the standard Wilson loop). $P_{\mu \nu}$ is a symmetrized plaquette in the $\mu-\nu$ plane, also denoted as a clover leaf. Equations (8) and (9) as well as the clover leaf are illustrated in Fig. 1.

\section{Angular dependence of flux densities}

As discussed in Sec. II A, for absolute total angular momentum $\Lambda \geq 1$, the spectrum is degenerate with respect to $\epsilon$, i.e., $V_{\Lambda_{\eta}^{+}}(r)=V_{\Lambda_{\eta}^{-}}(r)$. In other words, the states $\left|0_{\Lambda_{\eta}^{+}}(r)\right\rangle$ and $\left|0_{\Lambda_{\eta}^{-}}(r)\right\rangle$ have the same energy. Their flux densities $\Delta F_{j, \Lambda_{\eta}^{+}}^{2}(r ; \mathbf{x})$ and $\Delta F_{j, \Lambda_{\eta}^{-}}^{2}(r ; \mathbf{x})$ are, however, not identical but related by rotations, as we discuss in the following.

One can show that under rotations around the $z$ axis with angle $\alpha$ the states $\left|0_{\Lambda_{\eta}^{\varepsilon}}(r)\right\rangle$ transform according to

$$
R_{z}(\alpha)\left|0_{\Lambda_{\eta}^{ \pm}}(r)\right\rangle=\cos (\alpha \Lambda)\left|0_{\Lambda_{\eta}^{ \pm}}(r)\right\rangle+i \sin (\alpha \Lambda)\left|0_{\Lambda_{\eta}^{\mp}}(r)\right\rangle
$$

(see Appendix A), while the field strength components transform as

$$
\begin{aligned}
R_{z}^{\dagger}(\alpha) F_{j}^{a}(\mathbf{x}) R_{z}(\alpha) & =R_{j k}(-\alpha) F_{k}^{a}(R(-\alpha) \mathbf{x}) \\
& =R_{j k}(-\alpha) F_{k}^{a}\left(\mathbf{x}_{-\alpha}\right) .
\end{aligned}
$$

$R(\alpha)$ denotes the corresponding standard $3 \times 3$ rotation matrix, i.e., 


$$
R(\alpha)=\left(\begin{array}{ccc}
+\cos (\alpha) & -\sin (\alpha) & 0 \\
+\sin (\alpha) & +\cos (\alpha) & 0 \\
0 & 0 & 1
\end{array}\right),
$$

and we have defined $\mathbf{x}_{-\alpha}=R(-\alpha) \mathbf{x}$. Now, we consider the rotated flux densities $\left\langle 0_{\Lambda_{\eta}^{ \pm}}(r)\left|R_{z}^{\dagger}(\alpha) F_{j}^{2}(\mathbf{x}) R_{z}(\alpha)\right| 0_{\Lambda_{\eta}^{ \pm}}(r)\right\rangle-$ $\left\langle\Omega\left|F_{j}^{2}\right| \Omega\right\rangle$ and rewrite them in two different ways, using first Eq. (11),

$$
\begin{aligned}
\left\langle 0_{\Lambda_{\eta}^{ \pm}}\right. & \left.(r)\left|R_{z}^{\dagger}(\alpha) F_{j}^{2}(\mathbf{x}) R_{z}(\alpha)\right| 0_{\Lambda_{\eta}^{ \pm}}(r)\right\rangle-\left\langle\Omega\left|F_{j}^{2}\right| \Omega\right\rangle \\
= & \cos ^{2}(\alpha \Lambda) \Delta F_{j, \Lambda_{\eta}^{ \pm}}^{2}(r ; \mathbf{x})+\sin ^{2}(\alpha \Lambda) \Delta F_{j, \Lambda_{\eta}^{\mp}}^{2}(r ; \mathbf{x}) \\
& +i \cos (\alpha \Lambda) \sin (\alpha \Lambda)\left(\left\langle 0_{\Lambda_{\eta}^{ \pm}}(r)\left|F_{j}^{2}(\mathbf{x})\right| 0_{\Lambda_{\eta}^{\mp}}(r)\right\rangle\right. \\
& \left.-\left\langle 0_{\Lambda_{\eta}^{\mp}}(r)\left|F_{j}^{2}(\mathbf{x})\right| 0_{\Lambda_{\eta}^{ \pm}}(r)\right\rangle\right),
\end{aligned}
$$

then Eq. (12),

$$
\begin{aligned}
& \left\langle 0_{\Lambda_{\eta}^{ \pm}}(r)\left|R_{z}^{\dagger}(\alpha) F_{j}^{2}(\mathbf{x}) R_{z}(\alpha)\right| 0_{\Lambda_{\eta}^{ \pm}}(r)\right\rangle-\left\langle\Omega\left|F_{j}^{2}\right| \Omega\right\rangle \\
& =\left[\begin{array}{c}
c_{\alpha}^{2} \Delta F_{x, \Lambda_{\eta}^{ \pm}}^{2}\left(r ; \mathbf{x}_{-\alpha}\right)+s_{\alpha}^{2} \Delta F_{y, \Lambda_{\eta}^{ \pm}}^{2}\left(r ; \mathbf{x}_{-\alpha}\right)+2 c_{\alpha} s_{\alpha}\left\langle 0_{\Lambda_{\eta}^{ \pm}}(r)\left|F_{x}\left(\mathbf{x}_{-\alpha}\right) F_{y}\left(\mathbf{x}_{-\alpha}\right)\right| 0_{\Lambda_{\eta}^{ \pm}}(r)\right\rangle \\
c_{\alpha}^{2} \Delta F_{y, \Lambda_{\eta}^{ \pm}}^{2}\left(r ; \mathbf{x}_{-\alpha}\right)+s_{\alpha}^{2} \Delta F_{x, \Lambda_{\eta}^{ \pm}}^{2}\left(r ; \mathbf{x}_{-\alpha}\right)-2 c_{\alpha} s_{\alpha}\left\langle 0_{\Lambda_{\eta}^{ \pm}}(r)\left|F_{x}\left(\mathbf{x}_{-\alpha}\right) F_{y}\left(\mathbf{x}_{-\alpha}\right)\right| 0_{\Lambda_{\eta}^{ \pm}}(r)\right\rangle \\
\Delta F_{z, \Lambda_{\eta}^{ \pm}}^{2}\left(r ; \mathbf{x}_{-\alpha}\right)
\end{array}\right]_{j}
\end{aligned}
$$

with the shorthand notation $c_{\alpha}=\cos (\alpha)$ and $s_{\alpha}=\sin (\alpha)$ and where the index $j$ on the right-hand side indicates the $j$ th component of [...]. Equating Eqs. (14) and (15) relates the flux densities $\Delta F_{j, \Lambda_{\eta}^{e}}^{2}(r ; \mathbf{x})$ and $\Delta F_{j, \Lambda_{\eta}^{e}}^{2}\left(r ; \mathbf{x}_{-\alpha}\right)$, i.e., yields their transformation law with respect to rotations around the $z$ axis. ${ }^{1}$ Clearly, one cannot expect the flux densities $\Delta F_{j, \Lambda_{\eta}^{+}}^{2}(r ; \mathbf{x})$ and $\Delta F_{j, \Lambda_{\eta}^{-}}^{2}(r ; \mathbf{x})$ to be invariant under rotations, nor to appear to be identical, in particular not for $\Lambda \geq 1$, even though the corresponding potentials are degenerate. Numerical computations confirm that these flux densities are not invariant under rotations and that they are different from each other (see the discussion in Sec. IV B and the example plots in Figs. 5 and 6).

Instead of quantum numbers $\Lambda_{\eta}^{\epsilon}$, one can also use quantum numbers $\lambda_{\eta}$ to label hybrid static potential states with $\Lambda \geq 1$, where $\lambda=\ldots,-2,-1,+1,+2, \ldots$, is the total angular momentum with respect to the $z$ axis, i.e., $\Lambda=|\lambda|$. Of course, there are again the same pairs of degenerate potentials, i.e., $V_{+\lambda_{\eta}}(r)=V_{-\lambda_{\eta}}(r)=V_{\Lambda_{\eta}^{+}}(r)=V_{\Lambda_{\eta}^{-}}(r)$. In this case, the behavior of the corresponding states and flux densities under rotations is different,

$$
R_{z}(\alpha)\left|0_{\lambda_{\eta}}(r)\right\rangle=e^{i \alpha \lambda}\left|0_{\lambda_{\eta}}(r)\right\rangle
$$

and Eq. (14) simplifies,

$$
\begin{gathered}
\left\langle 0_{\lambda_{\eta}}(r)\left|R_{z}^{\dagger}(\alpha) F_{j}^{2}(\mathbf{x}) R_{z}(\alpha)\right| 0_{\lambda_{\eta}}(r)\right\rangle \\
-\left\langle\Omega\left|F_{j}^{2}\right| \Omega\right\rangle=\Delta F_{j, \lambda_{\eta}}^{2}(r ; \mathbf{x}),
\end{gathered}
$$

\footnotetext{
${ }^{1}$ Equations (14) and (15) simplify for cubic rotations and thus are very helpful to improve statistical precision by symmetrizing the lattice results accordingly (see Sec. IV B).
}

while Eq. (15) remains essentially unchanged (one just has to replace $\Lambda_{\eta}^{\epsilon}$ by $\lambda_{\eta}$ ). Consequently, the transformation law with respect to rotations around the $z$ axis and the angular dependence of $\Delta F_{j, \Lambda_{\eta}^{\epsilon}}^{2}(r ; \mathbf{x})$ and $\Delta F_{j, \lambda_{\eta}}^{2}(r ; \mathbf{x})$ is different, even though the corresponding hybrid static potentials are identical.

To eliminate this somewhat arbitrary angular dependence, which is a consequence of $\epsilon$ (when using quantum numbers $\Lambda_{\eta}^{\epsilon}$ ) or the sign of $\lambda$ (when using quantum numbers $\lambda_{\eta}$ ), but not related to $\Lambda=|\lambda|$ or $\eta[\Lambda$ and $\eta$ fully characterize hybrid static potentials $V_{\Lambda_{\eta}}(r)$ for $\Lambda \geq 1$, we define for $\Lambda \geq 1$

$$
\begin{aligned}
\Delta F_{j, \Lambda_{\eta}}^{2}(r ; \mathbf{x}) & =\frac{1}{2}\left(\Delta F_{j, \Lambda_{\eta}^{+}}^{2}(r ; \mathbf{x})+\Delta F_{j, \Lambda_{\eta}^{-}}^{2}(r ; \mathbf{x})\right) \\
& =\frac{1}{2} \operatorname{Tr}\left(\mathcal{P}_{\Lambda_{\eta}}\left(F_{j}^{2}(\mathbf{x})-\left\langle\Omega\left|F_{j}^{2}\right| \Omega\right\rangle\right)\right) .
\end{aligned}
$$

This quantity represents the average over an ensemble of states with fixed $\Lambda$ and $\eta$, but arbitrary $\epsilon$. After the last equality, the projector

$$
\begin{aligned}
\mathcal{P}_{\Lambda_{\eta}} & =\left|0_{\Lambda_{\eta}^{+}}(r)\right\rangle\left\langle 0_{\Lambda_{\eta}^{+}}(r)|+| 0_{\Lambda_{\eta}^{-}}(r)\right\rangle\left\langle 0_{\Lambda_{\eta}^{-}}(r)\right| \\
& =\left|0_{+\lambda_{\eta}}(r)\right\rangle\left\langle 0_{+\lambda_{\eta}}(r)|+| 0_{-\lambda_{\eta}}(r)\right\rangle\left\langle 0_{-\lambda_{\eta}}(r)\right|
\end{aligned}
$$

to the corresponding two-dimensional space of states has been introduced. This projector shows explicitly that $\Delta F_{j, \Lambda_{\eta}}^{2}(r ; \mathbf{x})$ is independent of the basis used for that two-dimensional space, i.e., independent of whether we use use $\epsilon$ or the sign of $\lambda$.

The transformation law with respect to rotations around the $z$ axis for $\Delta F_{j, \Lambda_{\eta}}^{2}(r ; \mathbf{x})$ is 


$$
\Delta F_{j, \Lambda_{\eta}}^{2}(r ; \mathbf{x})=\left[\begin{array}{c}
c_{\alpha}^{2} \Delta F_{x, \Lambda_{\eta}}^{2}\left(r ; \mathbf{x}_{-\alpha}\right)+s_{\alpha}^{2} \Delta F_{y, \Lambda_{\eta}}^{2}\left(r ; \mathbf{x}_{-\alpha}\right)+c_{\alpha} s_{\alpha} \sum_{\epsilon}\left\langle 0_{\Lambda_{\eta}^{\epsilon}}(r)\left|F_{x}\left(\mathbf{x}_{-\alpha}\right) F_{y}\left(\mathbf{x}_{-\alpha}\right)\right| 0_{\Lambda_{\eta}^{e}}(r)\right\rangle \\
c_{\alpha}^{2} \Delta F_{y, \Lambda_{\eta}}^{2}\left(r ; \mathbf{x}_{-\alpha}\right)+s_{\alpha}^{2} \Delta F_{x, \Lambda_{\eta}}^{2}\left(r ; \mathbf{x}_{-\alpha}\right)-c_{\alpha} s_{\alpha} \sum_{\epsilon}\left\langle 0_{\Lambda_{\eta}^{\epsilon}}(r)\left|F_{x}\left(\mathbf{x}_{-\alpha}\right) F_{y}\left(\mathbf{x}_{-\alpha}\right)\right| 0_{\Lambda_{\eta}^{e}}(r)\right\rangle \\
\Delta F_{z, \Lambda_{\eta}}^{2}\left(r ; \mathbf{x}_{-\alpha}\right)
\end{array}\right]_{j}
$$

where the left-hand side can be obtained by combining Eqs. (17), (18), and (19) and the right-hand side is essentially Eq. (15). To simplify this even further, it is convenient to define

$$
\Delta F_{\perp, \Lambda_{\eta}}^{2}(r ; \mathbf{x})=\frac{1}{2}\left(\Delta F_{x, \Lambda_{\eta}}^{2}(r ; \mathbf{x})+\Delta F_{y, \Lambda_{\eta}}^{2}(r ; \mathbf{x})\right),
$$

as, e.g., also done in a similar way in Ref. [51]. This quantity as well as $\Delta F_{z, \Lambda_{\eta}}^{2}(r ; \mathbf{x})$ are invariant under rotations around the $z$ axis, i.e.,

$$
\begin{aligned}
\Delta F_{\perp, \Lambda_{\eta}}^{2}(r ; \mathbf{x}) & =\Delta F_{\perp, \Lambda_{\eta}}^{2}\left(r ; \mathbf{x}_{-\alpha}\right), \\
\Delta F_{z, \Lambda_{\eta}}^{2}(r ; \mathbf{x}) & =\Delta F_{z, \Lambda_{\eta}}^{2}\left(r ; \mathbf{x}_{-\alpha}\right) .
\end{aligned}
$$

Similarly, for $\Lambda=0$,

$$
\begin{aligned}
\Delta F_{\perp, \Lambda_{\eta}^{\epsilon}}^{2}(r ; \mathbf{x}) & =\Delta F_{\perp, \Lambda_{\eta}^{\epsilon}}^{2}\left(r ; \mathbf{x}_{-\alpha}\right), \\
\Delta F_{z, \Lambda_{\eta}^{\epsilon}}^{2}(r ; \mathbf{x}) & =\Delta F_{z, \Lambda_{\eta}^{\epsilon}}^{2}\left(r ; \mathbf{x}_{-\alpha}\right),
\end{aligned}
$$

as can be read off from Eqs. (14) and (15).

\section{LATTICE SETUP}

The computations presented in this work have been performed using $\mathrm{SU}(2)$ and $\mathrm{SU}(3)$ lattice gauge theory. The corresponding gauge link configurations have been generated with the standard Wilson gauge action (see textbooks on lattice field theory, e.g., Ref. [53]). Since we are considering purely gluonic observables, we expect that there is little difference between our pure gauge theory results and corresponding results in full QCD. This expectation is supported by Ref. [20], where hybrid static potentials were computed both in pure gauge theory and QCD and no statistically significant differences were observed.

TABLE II. SU(2) and SU(3) gauge link ensembles.

\begin{tabular}{lcccc}
\hline \hline Gauge group & $\beta$ & $a$ in fm & $(L / a)^{3} \times T / a$ & $\begin{array}{c}\text { Number of } \\
\text { configurations }\end{array}$ \\
\hline $\mathrm{SU}(2)$ & 2.5 & 0.079 & $18^{4}$ & 13000 \\
$\mathrm{SU}(3)$ & 6.0 & 0.093 & $24^{4}$ & 48000 \\
\hline \hline
\end{tabular}

For the SU(2) simulations, we have used a standard heat bath algorithm. To eliminate correlations in Monte Carlo time, the gauge link configurations are separated by 100 heat bath sweeps. For the SU(3) simulations, we have used the Chroma QCD library [54]. There, the gauge link configurations are separated by 20 update sweeps, where each update sweep comprises a heat bath and four overrelaxation steps. Details of our simulated ensembles are collected in Table II, including the gauge coupling $\beta$, the lattice extent $(L / a)^{3} \times T / a$, and the number of gauge link configurations used for the flux tube computations. We also list the lattice spacing $a$ in femtometers, which is obtained by identifying $r_{0}$ with $0.5 \mathrm{fm}$ (see Refs. [31,55]). For the majority of computations for gauge group $\mathrm{SU}(2)$, we use the ensemble with $(L / a)^{3} \times T / a=24^{4}$. The ensemble with $(L / a)^{3} \times T / a=18^{4}$ is only used for exploring and excluding finite volume effects in Sec. IV A 3.

To improve the signal quality, standard smearing techniques are applied, when sampling $\tilde{W}$ appearing in Eqs. (8) and (9) and defined in Eq. (10):

(i) Spatial gauge links, i.e., links in $a_{S ; \Lambda_{\eta}^{e}}(-r / 2$, $\left.+r / 2 ; t_{0}\right)$ and $a_{S ; \Lambda_{\eta}^{e}}\left(-r / 2,+r / 2 ; t_{2}\right)$ [defined in Eq. (2)], are APE-smeared gauge links (for detailed equations, see, e.g., Ref. [56]), where the parameters $\alpha_{\mathrm{APE}}=0.5$ and $N_{\mathrm{APE}}=20$ have been optimized in Ref. [31] to generate large overlaps with the ground states $\left|0_{\Lambda_{\eta}^{\epsilon}}(r)\right\rangle^{2}{ }^{2}$ This allows one to identify plateaus in $\Delta F_{\text {eff } ; j, \Lambda_{\eta}^{\epsilon}}^{2}\left(r, t_{2}, t_{0} ; \mathbf{x}, t_{1}\right)$ at smaller temporal separations $t_{2}-t_{1}$ and $t_{1}-t_{0}$ [see Eq. (7)].

(ii) For certain computations, temporal gauge links, i.e., links in $U\left(+r / 2 ; t_{0}, t_{2}\right)$ and $U\left(-r / 2 ; t_{2}, t_{0}\right)$, are HYP2-smeared gauge links [57-59], which lead to a reduced self-energy of the static quarks and, consequently, to smaller statistical errors. This, however, introduces larger discretization errors for small $r$ as well as for $\mathbf{x}$ close to either $\mathbf{r}_{Q}=$ $(0,0,+r / 2)$ or $\mathbf{r}_{\bar{Q}}=(0,0,-r / 2)$. Therefore, we use HYP2-smearing only, when computing field strengths $\Delta F_{j, \Lambda_{\eta}^{\epsilon}}^{2}(r ; \mathbf{x}=(x, y, 0))$ [see Eq. (7)], i.e., in the mediator plane $z=0$. For a more detailed discussion, see Sec. IV A 2.

\footnotetext{
${ }^{2}$ The optimzation of APE-smearing parameters in Ref. [31] was done for SU(3) gauge theory. We use the same parameters for our computations in SU(2) gauge theory and get similar ground state overlaps, which is indicated by effective mass plateaus of approximately the same quality.
} 
All statistical errors shown and quoted throughout this paper are determined via a jackknife. We perform a suitable binning of gauge link configurations to exclude statistical correlations in Monte Carlo time.

\section{NUMERICAL RESULTS}

\section{A. Investigation of systematic errors}

\section{Plateaus of $\Delta F_{\mathrm{eff} ; j, \Lambda_{n}^{\epsilon}}^{2}$ and contamination by excited states}

We have determined $\Delta F_{j, \Lambda_{\eta}^{e}}^{2}(r ; \mathbf{x})$ by fitting a constant to the lattice result for $\Delta F_{\text {eff } ; j, \Lambda_{\eta}^{\epsilon}}^{2}\left(r, t_{2}, t_{0} ; \mathbf{x}, t_{1}\right)$ at sufficiently large $t_{2}-t_{1}$ and $t_{1}-t_{0}$, where the data points are consistent with a plateau [see Eqs. (7), (8), and (9)]. For even $\left(t_{2}-t_{0}\right) / a$, we use $t_{1}=\left(t_{0}+t_{2}\right) / 2$, while for odd $\left(t_{2}-t_{0}\right) / a$, we use $t_{1}=\left(t_{0}+t_{2}+a\right) / 2$, i.e., equal or similar values for $t_{2}-t_{1}$ and $t_{1}-t_{0}$. Example plots of $\Delta F_{\text {eff } ; j, \Lambda_{\eta}^{e}}^{2}\left(r, t_{2}, t_{0} ; \mathbf{x}, t_{1}\right)$ as a function of $t_{2}-t_{0}$ for gauge group $\mathrm{SU}(2)$, all investigated $\Lambda_{\eta}^{\epsilon}$ sectors, quark-antiquark separation $r=10 a$, and $\mathbf{x}=\overrightarrow{0}$ are shown in Fig. 2 . We have performed an uncorrelated $\chi^{2}$-minimizing fit of a constant corresponding to $\Delta F_{j, \Lambda_{\eta}^{e}}^{2}(r ; \mathbf{x})$ in the region $t_{\min } \leq t_{2}-t_{0} \leq t_{\max }$. Since the statistical errors of $\Delta F_{\mathrm{eff} ; j, \Lambda_{\eta}^{\epsilon}}^{2}\left(r, t_{2}, t_{0} ; \mathbf{x}, t_{1}\right)$ rapidly increase with increasing $t_{2}-t_{0}$, the results are almost independent of $t_{\max }$. We have taken the largest $t_{\max }$, where the signal is not lost in noise. $t_{\text {min }}$ has been chosen such that $\chi^{2} /$ dof $\lesssim 1$ for the majority of fits. This results in $t_{\min } \approx 3 a \ldots 4 a$ and $t_{\max } \approx 5 a \ldots 8 a$ for hybrid static potentials with $\Lambda_{\eta}^{\epsilon}=\Sigma_{u}^{+}, \Sigma_{g}^{-}, \Sigma_{u}^{-}, \Pi_{g}, \Pi_{u}$, $\Delta_{g}, \Delta_{u}$, while $t_{\min } \approx 5 a \ldots 6 a$ and $t_{\max }=10 a$ for the ordinary static potential with $\Lambda_{\eta}^{\epsilon}=\Sigma_{g}^{+}$.

As an additional check that $t_{\min }$ is chosen sufficiently large, i.e., that excited states are strongly suppressed, we have repeated the computation of $\Delta F_{j, \Lambda_{\eta}^{e}}^{2}(r ; \mathbf{x})$ for gauge group $\mathrm{SU}(2), \Lambda_{\eta}^{\epsilon}=\Pi_{u}, r=6 a$, and $\mathbf{x}=(x, 0,0)$ using a creation operator $S$ [see Eq. (2)], which has a structure significantly different from that shown in Table I, namely, $S_{\mathrm{I}, 1}$ as defined in Ref. [31], Fig. 2. Within statistical errors, we find identical flux densities $\Delta F_{j, \Lambda_{\eta}^{\varepsilon}}^{2}(r ; \mathbf{x})$, which we interpret as confirmation that we indeed measure the flux densities of the ground states in the $\Lambda_{\eta}^{\epsilon}$ sectors and not flux densities, which depend on the creation operators and trial states we are using.

\section{Discretization errors and smearing}

Until now, we have performed computations only at a single value of the lattice spacing $a$. Therefore, we are not yet able to study the continuum limit. Strong discretization errors are expected, when either $r=\left|\mathbf{r}_{Q}-\mathbf{r}_{\bar{Q}}\right|,\left|\mathbf{x}-\mathbf{r}_{Q}\right|$ or $\left|\mathbf{x}-\mathbf{r}_{\bar{Q}}\right|$ is small, where $\mathbf{r}_{Q}=(0,0,+r / 2)$ and $\mathbf{r}_{\bar{Q}}=$ $(0,0,-r / 2)$ are the positions of the static charges and $\mathbf{x}$ is the spatial argument of the flux density $\Delta F_{j, \Lambda_{\eta}^{e}}^{2}(r ; \mathbf{x})$. These discretization errors are expected to be even more pronounced when using HYP2-smeared temporal links in $\tilde{W}$ [see Eq. (10)], which can be interpreted as increasing the radii of the static charges. We therefore compare results for $\Delta F_{j, \Lambda_{\eta}^{e}}^{2}(r ; \mathbf{x})$ obtained with and without HYP2-smeared temporal links.

In Fig. 3, we show results for $\Lambda_{\eta}^{\epsilon}=\Sigma_{g}^{+}, \Sigma_{u}^{-}$and $Q \bar{Q}$ separation $r=10 a$ on the separation axis, $\mathbf{x}=(0,0, z)$. For $\left|\mathbf{x}-\mathbf{r}_{Q}\right| \leq a$ or $\left|\mathbf{x}-\mathbf{r}_{\bar{Q}}\right| \leq a$, drastic discrepancies between unsmeared and HYP2-smeared results can be observed, while for $\left|\mathbf{x}-\mathbf{r}_{Q}\right|, \quad\left|\mathbf{x}-\mathbf{r}_{\bar{Q}}\right| \geq 4 a$ and $\Delta F_{j, \Lambda_{\eta}^{e}}^{2}(r ; \mathbf{x})=\Delta E_{z, \Lambda_{\eta}^{\epsilon}}^{2}(r ; \mathbf{x})$ as well as for $\left|\mathbf{x}-\mathbf{r}_{Q}\right|$, $\left|\mathbf{x}-\mathbf{r}_{\bar{Q}}\right| \geq 3 a$ and all other field strength components, there is agreement within statistical errors. When using HYP2-smeared temporal links, the pronounced peaks at the positions of the charges, which are present in the unsmeared results, are essentially gone. This is expected and can be observed in a qualitatively similar way also in much simpler theories, for example in classical electrodynamics, when smearing the charge density of a point charge. Analogous plots for other $\Lambda_{\eta}^{\epsilon}$ sectors look very similar and are not shown. Therefore, for the computations of $\Delta F_{j, \Lambda_{\eta}^{c}}^{2}(r ; \mathbf{x})$ in a plane containing the separation axis (see Sec. IV C), we do not use HYP2 smearing. Note, however, that even unsmeared results within a radius of about $2 a$ around either of the two static charges will exhibit sizable discretization errors and should be considered as crude estimates only. In other words, instead of the poles related to the infinite self-energy of the static charges, $\Delta F_{j, \Lambda_{\eta}^{\epsilon}}^{2}(r ; \mathbf{x})$ will exhibit pronounced but finite peaks.

We also study the effect of HYP2 smearing on $\Delta F_{j, \Lambda_{\eta}^{e}}^{2}(r ; \mathbf{x})$ in the mediator plane defined by $z=0$ for various $Q \bar{Q}$ separations $r$. For $r \geq 6 a$, we find agreement within statistical errors for all $\Lambda_{\eta}^{\epsilon}$ sectors and all field strength components with the exception of $\Delta E_{z, \Lambda_{\eta}^{e}}^{2}(r ; \mathbf{x}=(x, y, 0))$. For $\Delta E_{z, \Lambda_{\eta}^{e}}^{2}(r ; \mathbf{x}=(x, y, 0))$, there is agreement for $r \geq 10 a$ for $\Lambda_{\eta}^{\epsilon}=\Sigma_{g}^{+}$and for $r \geq 8 a$ for all other $\Lambda_{\eta}^{\epsilon}$ sectors. Example plots for $\Lambda_{\eta}^{\epsilon}=\Sigma_{g}^{+}, \Sigma_{u}^{-}$and $\mathbf{x}=(0,0,0)$ are shown in Fig. 4 . Therefore, for computations of $\Delta F_{j, \Lambda_{\eta}^{e}}^{2}$ in the mediator plane, which we show for $r=10 a$ in Sec. IV C, we use HYP2 smearing, which reduces statistical errors significantly.

\section{Finite volume corrections}

Finite volume corrections are rather mild for static potentials, when $r<L / 2$, where $L$ is the spatial lattice extent. In particular, for pure gauge theory, where the lightest particle (the $J^{\mathcal{P C}}=0^{++}$glueball) is very heavy, finite volume corrections should be almost negligible. A comparison of flux densities $\Delta F_{j, \Lambda_{\eta}^{e}}^{2}(r ; \mathbf{x})$ for gauge group $\mathrm{SU}(2)$ on the two gauge link ensembles with 


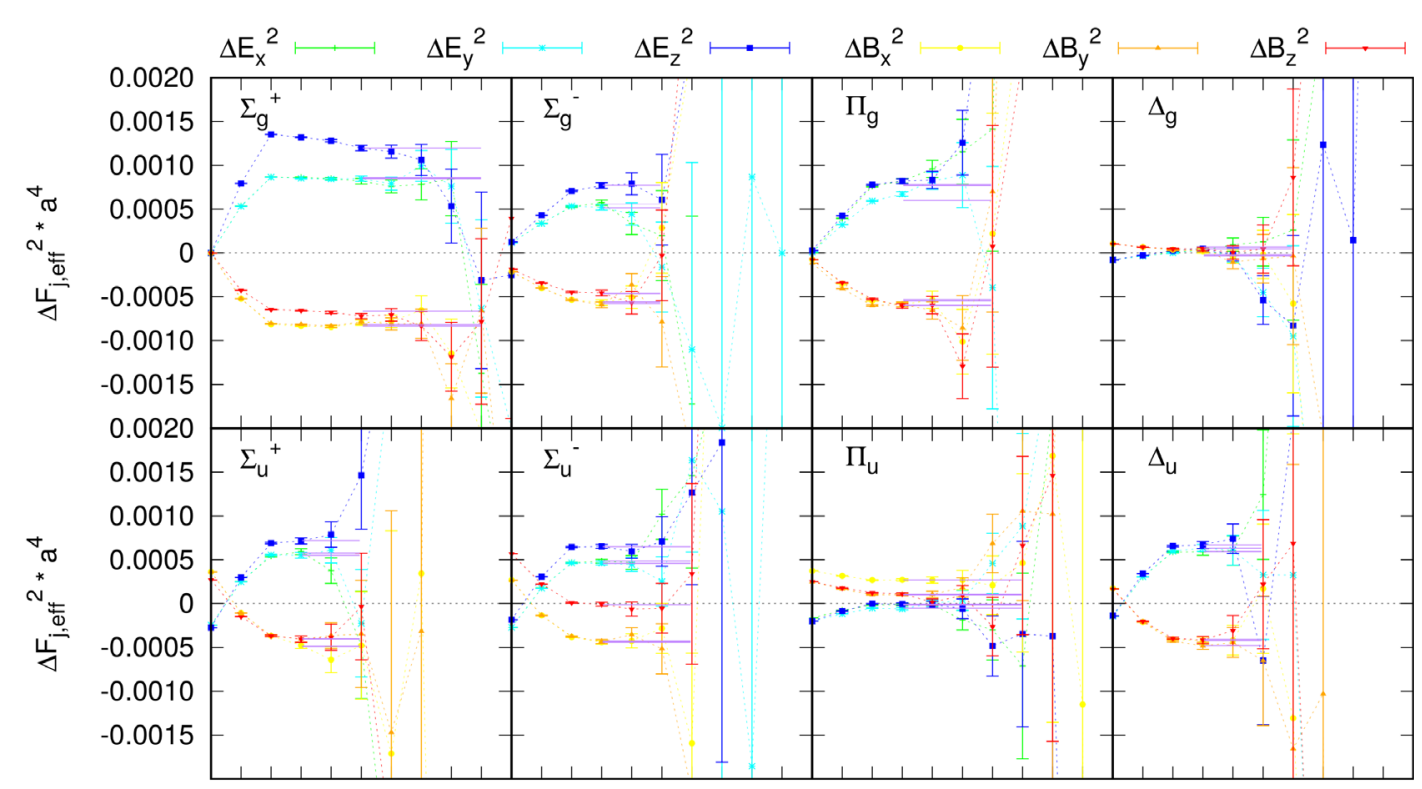

0123456789012345678901234567890123456789

$$
\left(\begin{array}{lll}
\left.t_{2}-t_{0}\right) / a & \left(t_{2}-t_{0}\right) / a & \left(t_{2}-t_{0}\right) / a
\end{array}\right.
$$

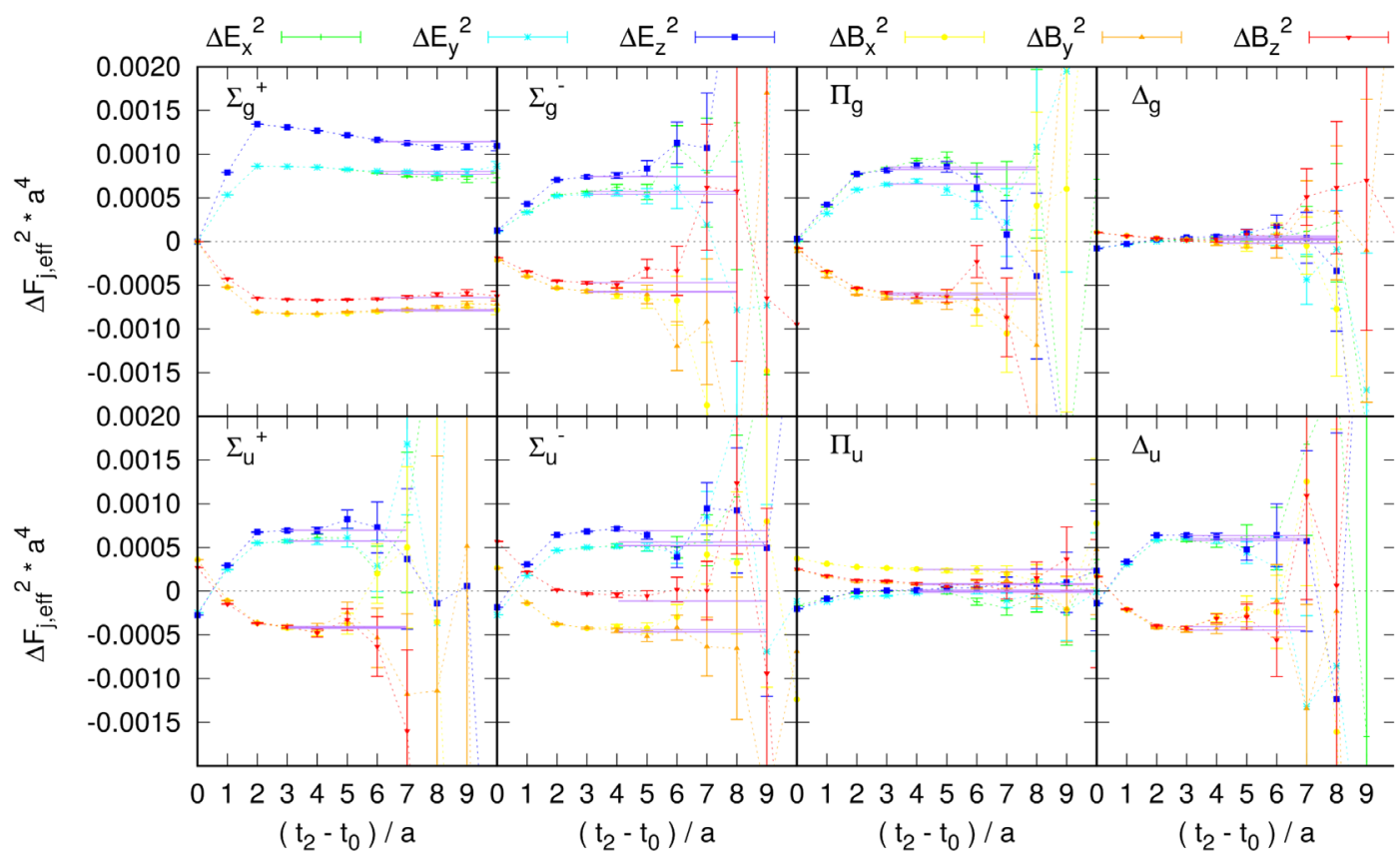

FIG. 2. $\Delta F_{\text {eff; } ;, \Lambda_{\eta}^{\epsilon}}^{2}\left(r, t_{2}, t_{0} ; \mathbf{x}=\overrightarrow{0}, t_{1}\right)$ as a function of $t_{2}-t_{0}$ for gauge group $\operatorname{SU}(2) ; \Lambda_{\eta}^{\epsilon}=\Sigma_{g}^{+}, \Sigma_{u}^{+}, \Sigma_{g}^{-}, \Sigma_{u}^{-}, \Pi_{g}, \Pi_{u}, \Delta_{g}, \Delta_{u}$; and $Q \bar{Q}$ separation $r=10 a$. Plateau fits and fitting ranges $\left[t_{\min }, t_{\max }\right]$ are indicated by horizontal straight lines. (Top) Temporal links in $\tilde{W}$ are unsmeared. (Bottom) Temporal links in $\tilde{W}$ are HYP2 smeared.

$(L / a)^{3} \times T / a=18^{4} \quad$ and $\quad(L / a)^{3} \times T / a=24^{4} \quad$ (see Table II) supports this expectation.

\section{B. Angular dependence and symmetrization of hybrid static potential flux densities}

In Sec. II B, we have discussed how hybrid static potential flux densities transform under rotations around the $z$ axis. On a hypercubic lattice, the relevant Eqs. (14) and (15) are exact only for cubic rotations, i.e., for rotations with angle $\alpha$, which is a multiple of $\pi / 2$. For $\alpha= \pm \pi / 2$, they become for even $\Lambda$, i.e., $\Lambda=\Sigma$ and $\Lambda=\Delta$,

$$
\begin{aligned}
& \Delta F_{x, \Lambda_{\eta}^{ \pm}}^{2}(r ; \mathbf{x})=\Delta F_{y, \Lambda_{\eta}^{ \pm}}^{2}\left(r ; \mathbf{x}_{ \pm \pi / 2}\right) \\
& \Delta F_{y, \Lambda_{\eta}^{ \pm}}^{2}(r ; \mathbf{x})=\Delta F_{x, \Lambda_{\eta}^{ \pm}}^{2}\left(r ; \mathbf{x}_{ \pm \pi / 2}\right) \\
& \Delta F_{z, \Lambda_{\eta}^{ \pm}}^{2}(r ; \mathbf{x})=\Delta F_{z, \Lambda_{\eta}^{ \pm}}^{2}\left(r ; \mathbf{x}_{ \pm \pi / 2}\right),
\end{aligned}
$$

and for odd $\Lambda$, i.e., $\Lambda=\Pi$, 


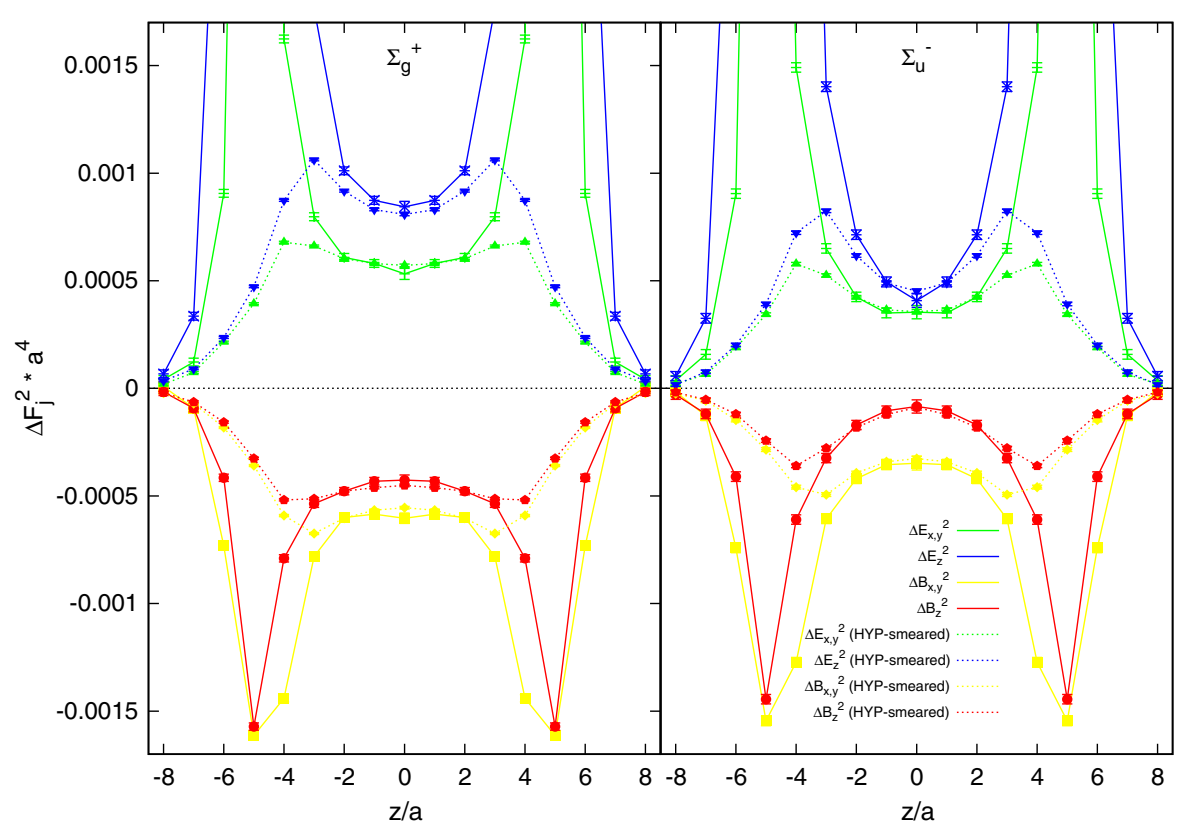

FIG. 3. Flux densities $\Delta F_{j, \Lambda_{\eta}^{\epsilon}}^{2}(r ; \mathbf{x}=(0,0, z))$ as a function of $z$ for gauge group $\mathrm{SU}(3), \Lambda_{\eta}^{\epsilon}=\Sigma_{g}^{+}, \Sigma_{u}^{-}$and $Q \bar{Q}$ separation $r=10 a$.

$$
\begin{aligned}
& \Delta F_{x, \Lambda_{\eta}^{ \pm}}^{2}(r ; \mathbf{x})=\Delta F_{y, \Lambda_{\eta}^{\mp}}^{2}\left(r ; \mathbf{x}_{ \pm \pi / 2}\right) \\
& \Delta F_{y, \Lambda_{\eta}^{ \pm}}^{2}(r ; \mathbf{x})=\Delta F_{x, \Lambda_{\eta}^{\mp}}^{2}\left(r ; \mathbf{x}_{ \pm \pi / 2}\right) \\
& \Delta F_{z, \Lambda_{\eta}^{ \pm}}^{2}(r ; \mathbf{x})=\Delta F_{z, \Lambda_{\eta}^{\mp}}^{2}\left(r ; \mathbf{x}_{ \pm \pi / 2}\right) .
\end{aligned}
$$

We have verified our numerical computation of flux densities using these equations; i.e., we have checked that all our results are consistent with these equations within statistical errors. In a second step, we have used these equations to reduce the statistical errors of our results by averaging related flux densities.

In Sec. II B, we have also discussed that hybrid static potential flux densities $\Delta F_{j, \Lambda_{\eta}^{+}}^{2}(r ; \mathbf{x})$ and $\Delta F_{j, \Lambda_{\eta}^{-}}^{2}(r ; \mathbf{x})$ with $\Lambda \geq 1$ are not expected to be identical, even though the corresponding potentials are degenerate [see Eqs. (14) and (15)]. This expectation is confirmed by the plots in the

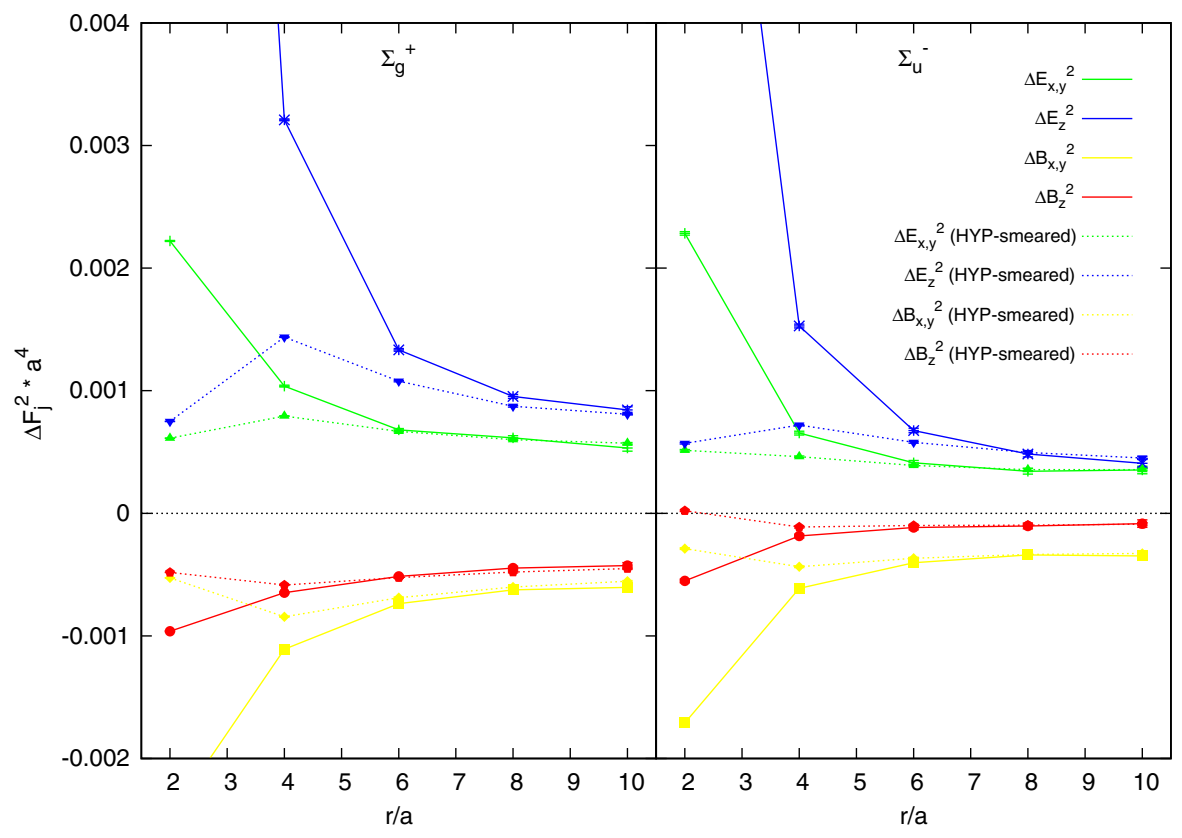

FIG. 4. Flux densities $\Delta F_{j, \Lambda_{\eta}^{\epsilon}}^{2}(r ; \mathbf{x}=\overrightarrow{0})$ as a function of the $Q \bar{Q}$ separation $r$ for gauge group $\mathrm{SU}(3)$ and $\Lambda_{\eta}^{\epsilon}=\Sigma_{g}^{+}, \Sigma_{u}^{-}$. 

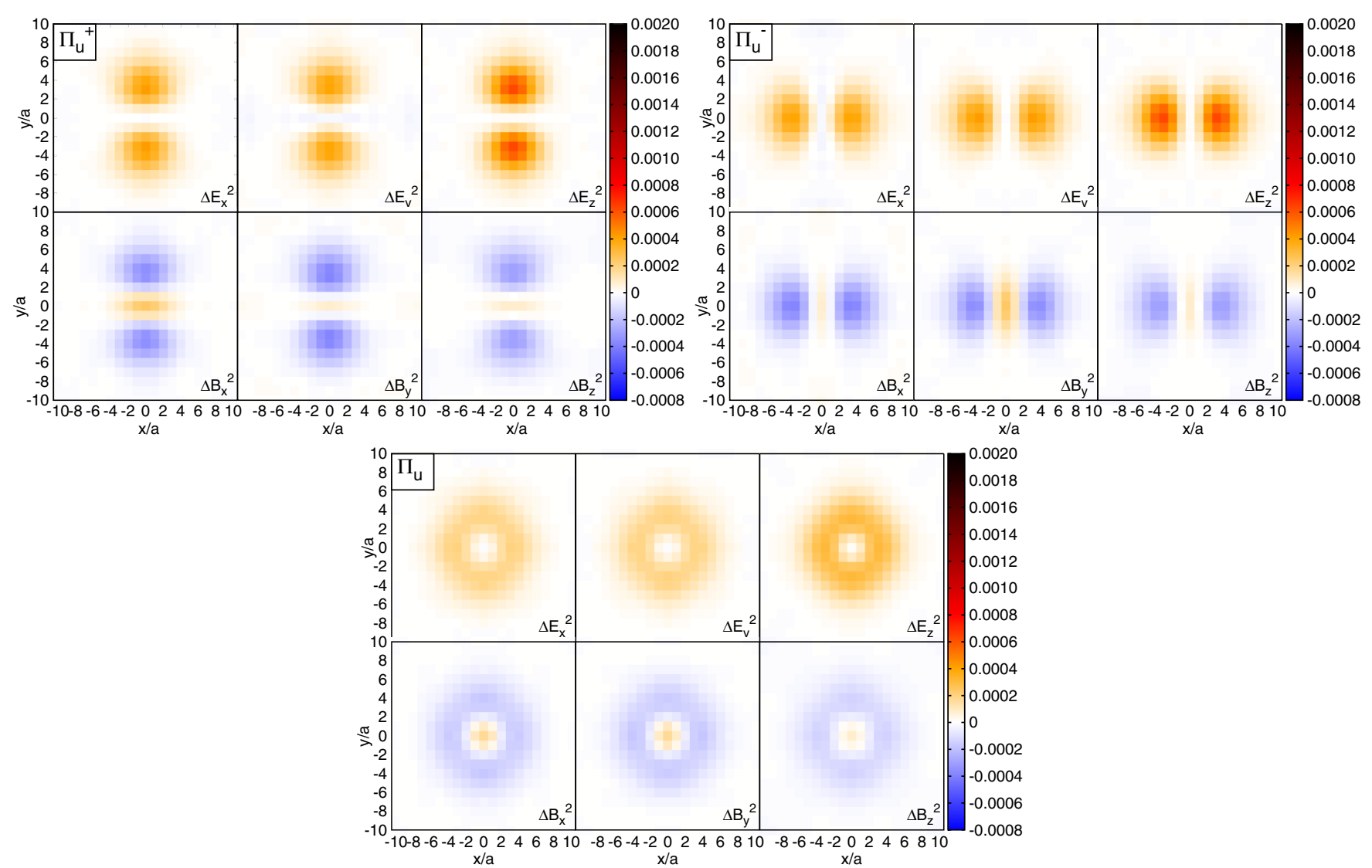

FIG. 5. Flux densities $\Delta F_{j, \Pi_{u}^{+}}^{2}(r ; \mathbf{x}), \Delta F_{j, \Pi_{\bar{u}}^{-}}^{2}(r ; \mathbf{x})$, and $\Delta F_{j, \Pi_{u}}^{2}(r ; \mathbf{x})$ in the mediator plane $(z=0)$ for gauge group $\mathrm{SU}(2)$ and $Q \bar{Q}$ separation $r=10 a$.

upper rows of Figs. 5 and 6, where we show two examples, the flux densities $\Delta F_{j, \Lambda_{\eta}^{e}}^{2}(r ; \mathbf{x})$ for $\Lambda_{\eta}^{\epsilon}=\Pi_{u}^{+}, \Pi_{u}^{-}$and for $\Lambda_{\eta}^{\epsilon}=\Delta_{g}^{+}, \Delta_{g}^{-}$in the mediator plane $z=0$.

In the plots at the bottom of Figs. 5 and 6, we show the flux densities $\Delta F_{j, \Lambda_{\eta}}^{2}(r ; \mathbf{x})$ defined in Eq. (18), again for $\Lambda_{\eta}=\Pi_{u}$ and for $\Lambda_{\eta}=\Delta_{g}$. As discussed in Sec. II B, these are ensemble averages over states with fixed $\Lambda$ and $\eta$, but indefinite $\epsilon$. Note that $\Delta F_{z, \Lambda_{\eta}}^{2}(r ; \mathbf{x})$ is invariant under cubic rotations, while $\Delta F_{x, \Lambda_{\eta}}^{2}(r ; \mathbf{x})$ and $\Delta F_{y, \Lambda_{\eta}}^{2}(r ; \mathbf{x})$, even though quite similar, are related by rotations with angle $\alpha= \pm \pi / 2$ [see Eq. (20)]. Averaging $\Delta F_{x, \Lambda_{\eta}}^{2}(r ; \mathbf{x})$ and $\Delta F_{y, \Lambda_{\eta}}^{2}(r ; \mathbf{x})$ according to Eq. (21) would lead to another quantity invariant under cubic rotations. From now on we always show the flux densities $\Delta F_{j, \Lambda_{\eta}}^{2}(r ; \mathbf{x})$ for $\Lambda \geq 1$, i.e., not anymore $\Delta F_{j, \Lambda_{\eta}^{e}}^{2}(r ; \mathbf{x})$.

\section{Hybrid static potential flux densities for all $\Lambda_{\eta}^{\epsilon}$ sectors}

In this section, we show and discuss the main numerical results of this work, the flux densities $\Delta F_{j, \Lambda_{\eta}^{(e)}}^{2}(r ; \mathbf{x})$, $j=x, y, z, \perp$ for the eight sectors $\Lambda_{\eta}^{(\epsilon)}=\Sigma_{g}^{+}, \Sigma_{g}^{-}, \Sigma_{u}^{+}, \Sigma_{u}^{-}$,
$\Pi_{g}, \Pi_{u}, \Delta_{g}, \Delta_{u}$, both in the mediator plane $z=0$ and in the separation plane $y=0$. All plots in this section are for $\mathrm{SU}(2)$ gauge theory. Corresponding plots for SU(3) gauge theory are very similar and collected in Appendix B.

We decided to perform computations for two $Q \bar{Q}$ separations, $r=6 a$ and $r=10 a$. This allows us to compare results for two significantly different $r$, i.e., to see how the shapes of the hybrid static potential flux tubes change, when the quark and the antiquark are pulled apart. We did not study separations $r<6 a$ because for such small separations flux densities exhibit sizable discretization errors in the region between the two charges (see the discussion in Sec. IVA 2). Since the signal for a Wilson loop decays exponentially with its area, we also refrained from performing computations for $r>10 a$, which are very costly in terms of CPU time.

Since the resulting flux densities in the mediator plane for $r=6 a$ and $r=10 a$ are very similar, we only present them for $r=10 a$. In Fig. 7, these flux densities $\Delta F_{j, \Lambda_{\eta}^{(\epsilon)}}^{2}(r ; \mathbf{x}=(x, y, 0)), j=x, y, z$ are shown as twodimensional (2D) color maps. In the upper panel of Fig. 8, we present similar results, the rotationally invariant $\Delta F_{j, \Lambda_{\eta}^{(\epsilon)}}^{2}(r ; \mathbf{x}=(x, 0,0)), j=\perp, z$ along the $x$ axis, i.e., in 

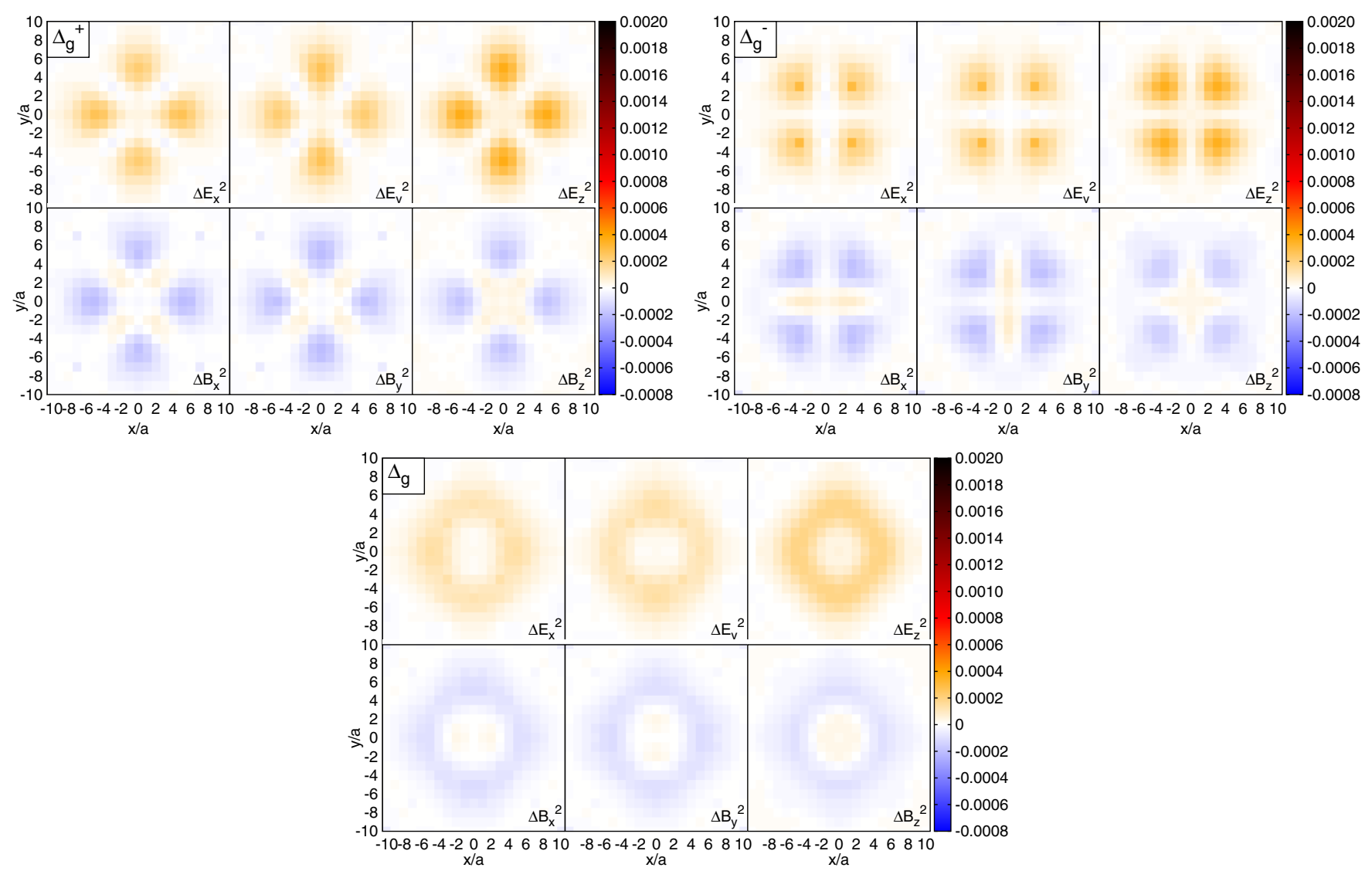

FIG. 6. Flux densities $\Delta F_{j, \Delta_{g}^{+}}^{2}(r ; \mathbf{x}), \Delta F_{j, \Delta_{g}^{-}}^{2}(r ; \mathbf{x})$, and $\Delta F_{j, \Delta_{g}}^{2}(r ; \mathbf{x})$ in the mediator plane $(z=0)$ for gauge group SU(2) and $Q \bar{Q}$ separation $r=10 a$.

the mediator plane as a function of the radial coordinate. In contrast to the 2D color maps, these one-dimensional (1D) curves allow us to also show statistical errors and thus provide information about the precision of our numerical results. In the lower panel of Fig. 8, we present in the same style differences of hybrid static potential flux densities as those of the ordinary static potential, i.e., $\Delta F_{j, \Lambda_{\eta}^{(e)}}^{2}(r ; \mathbf{x}=(x, 0,0))-\Delta F_{j, \Sigma_{g}^{+}}^{2}(r ; \mathbf{x}=$ $(x, 0,0)), j=\perp, z$. Flux densities $\Delta F_{j, \Lambda_{\eta}^{(e)}}^{2}(r ; \mathbf{x}=(x, 0, z))$, $j=x, y, z$ in the separation plane are shown as $2 \mathrm{D}$ color maps in Figs. 9 and 10 for both separations $r=6 a$ and $r=10 a$. Note that flux densities close to one of the static charges, in particular for $\left|\mathbf{x}-\mathbf{r}_{Q}\right| \leq a$ or $\left|\mathbf{x}-\mathbf{r}_{\bar{Q}}\right| \leq a$, exhibit sizable discretization errors (see the discussion in Sec. IVA 2).

The flux densities of the ordinary static potential form a cigar-shaped flux tube with strong positive contributions to the energy density from the chromoelectric and smaller negative contributions from the chromomagnetic field strength components. The maxima are on the $Q \bar{Q}$ separation axis, i.e., at $x=y=0$. While this is known from previous lattice gauge theory investigations of the ordinary static potential (see, e.g., Ref. [39]), the corresponding flux densities for hybrid static potentials show a variety of different and interesting structures. For example, chromomagnetic flux densities of hybrid static potentials are typically larger close to the center of the flux tube than those of the ordinary static potential, as can be seen in Fig. 8, lower panel. Hybrid static potential flux tubes are also wider, i.e., have a larger extension in the $x$ and $y$ directions (see, e.g., Figs. 8, 9, and 10). Another interesting difference is that some hybrid static potentials show a clear reduction of the chromoelectric flux densities close to the center, while the chromomagnetic flux densities exhibit peaks (most prominently for $\Lambda_{\eta}=\Pi_{u}, \Delta_{g}$, but to some extent also for $\left.\Lambda_{\eta}^{\epsilon}=\Sigma_{u}^{-}\right)$. For other sectors, $\Lambda_{\eta}^{(\epsilon)}=\Sigma_{u}^{+}, \Pi_{g}, \Delta_{u}$, the opposite is the case; i.e., there is a positive localized peak at the center for the chromoelectric flux densities and a corresponding negative contribution of the chromomagnetic flux densities. These peaks in either the chromoelectric or chromomagnetic flux densities can be interpreted as "valence gluons" generating the hybrid quantum numbers, as discussed in models and phenomenological descriptions of hybrid mesons. The positive or negative peaks are surrounded by spherical shells, where flux densities are smaller or larger, respectively 

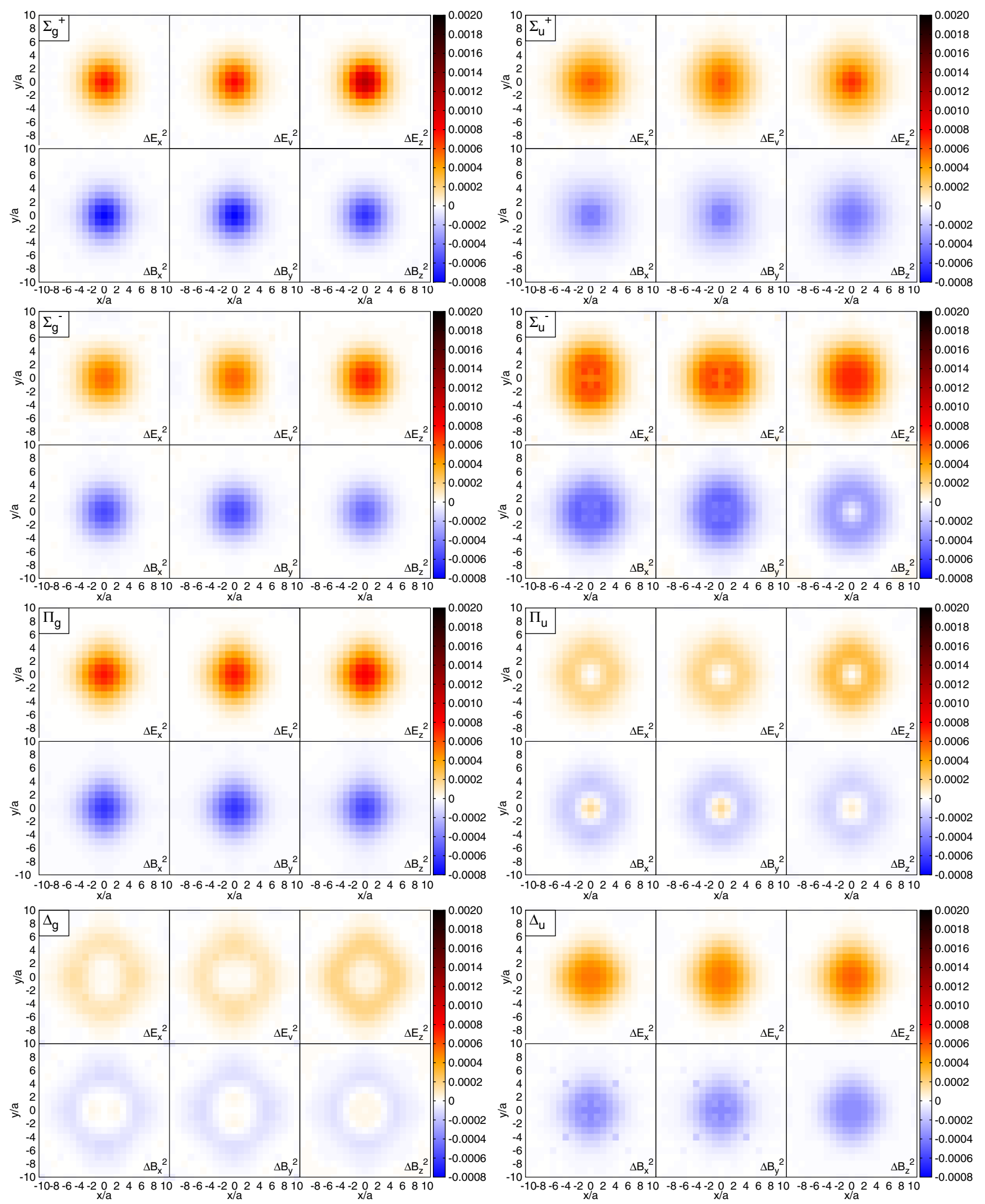

FIG. 7. Flux densities $\left.\Delta F^{2}{ }_{(\epsilon)}^{(\epsilon ;} ; \mathbf{x}=(x, y, 0)\right), j=x, y, z$ in the mediator plane for gauge group $\mathrm{SU}(2)$, all investigated sectors $\Lambda_{\eta}^{(e)}=\Sigma_{g}^{+}, \Sigma_{u}^{+}, \Sigma_{g}^{-}, \Sigma_{u}^{-}, \Pi_{g}, \Pi_{u}^{j, \Lambda_{\eta}}, \Delta_{g}, \Delta_{u}$ and $Q \bar{Q}$ separation $r=10 a$. 

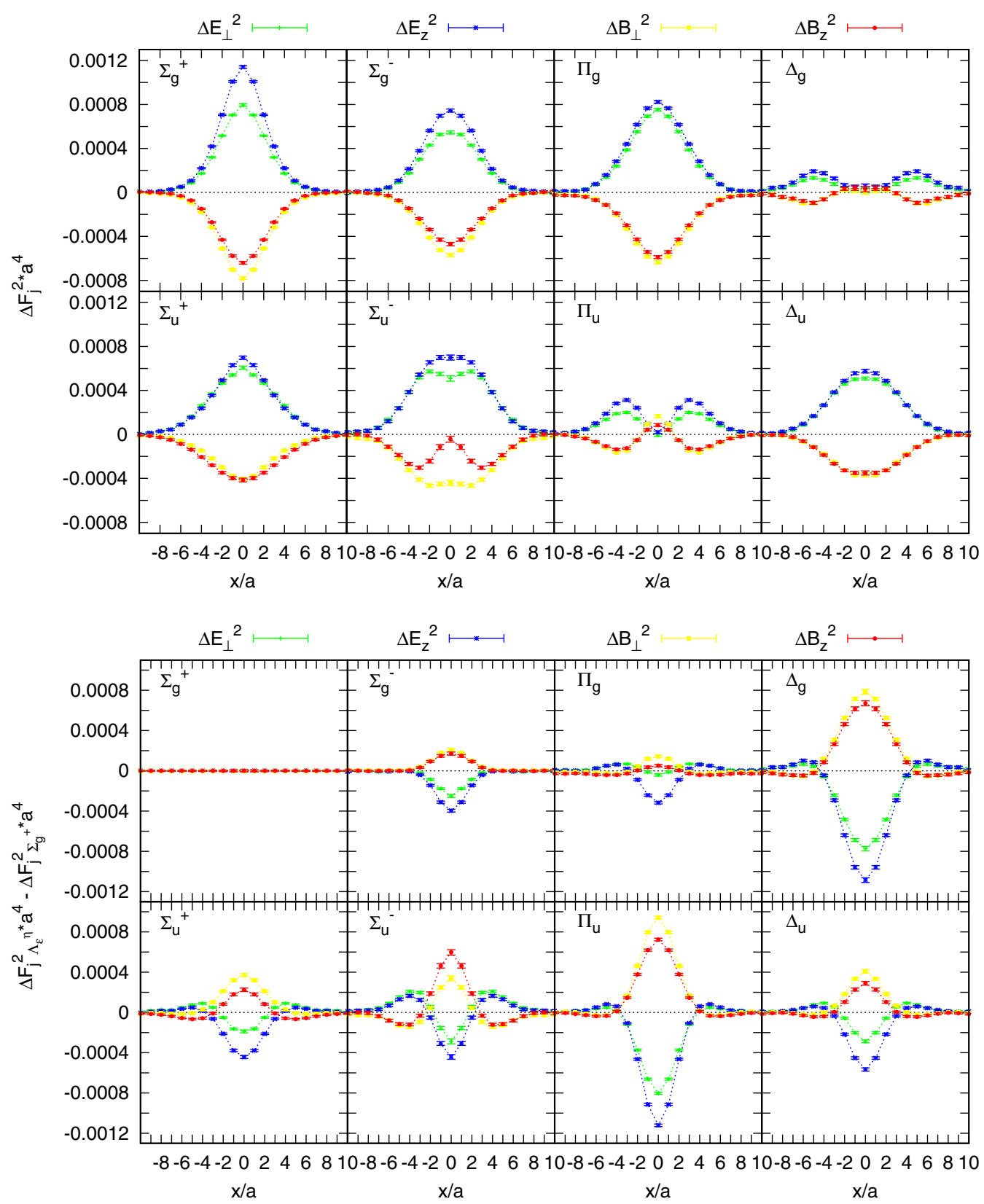

FIG. 8. Flux densities on the $x$ axis for gauge group $\mathrm{SU}(2)$, all investigated sectors $\Lambda_{\eta}^{(\epsilon)}=\Sigma_{g}^{+}, \Sigma_{u}^{+}, \Sigma_{g}^{-}, \Sigma_{u}^{-}, \Pi_{g}, \Pi_{u}, \Delta_{g}, \Delta_{u}$ and $Q \bar{Q}$ separation $r=10 a$. (Top) $\Delta F_{j, \Lambda_{\eta}^{(c)}}^{2}(r ; \mathbf{x}=(x, 0,0)), j=\perp, z$. (Bottom) $\Delta F_{j, \Lambda_{\eta}^{(c)}}^{2}(r ; \mathbf{x}=(x, 0,0))-\Delta F_{j, \Sigma_{g}^{+}}^{2}(r ; \mathbf{x}=(x, 0,0)), j=\perp, z$.

(see in particular the 2D color maps in Figs. 9 and 10, where these shells are visible as rings). These structures remind us of and might indicate vibrating strings, which have either nodes or maxima at $z=0$. Moreover, the transverse extent of the structures formed by the chromoelectric or chromomagnetic flux densities is almost the same for $Q \bar{Q}$ separation $r=6 a$ and $r=10 a$, which is consistent with a string interpretation. One should, however, keep in mind that our $Q \bar{Q}$ separations correspond to 0.47 and $0.79 \mathrm{fm}$, respectively, which is rather small, while an effective string theory description of flux tubes is expected to be valid for $r \gtrsim 2 / \sqrt{\sigma} \approx 1 \mathrm{fm}$ (see, e.g., Ref. [60]). Thus, it would be interesting to perform similar computations at larger $Q \bar{Q}$ separations and to compare such results in more detail to effective string theory predictions.

It is also interesting to compare the resulting flux densities to the gluonic excitation operators for hybrid static potentials at leading order in the multipole expansion of potential Non-Relativistic QCD (pNRQCD) (see, e.g., Refs. [2,61]). Similar operators were also used in lattice gauge theory computations of hybrid static 

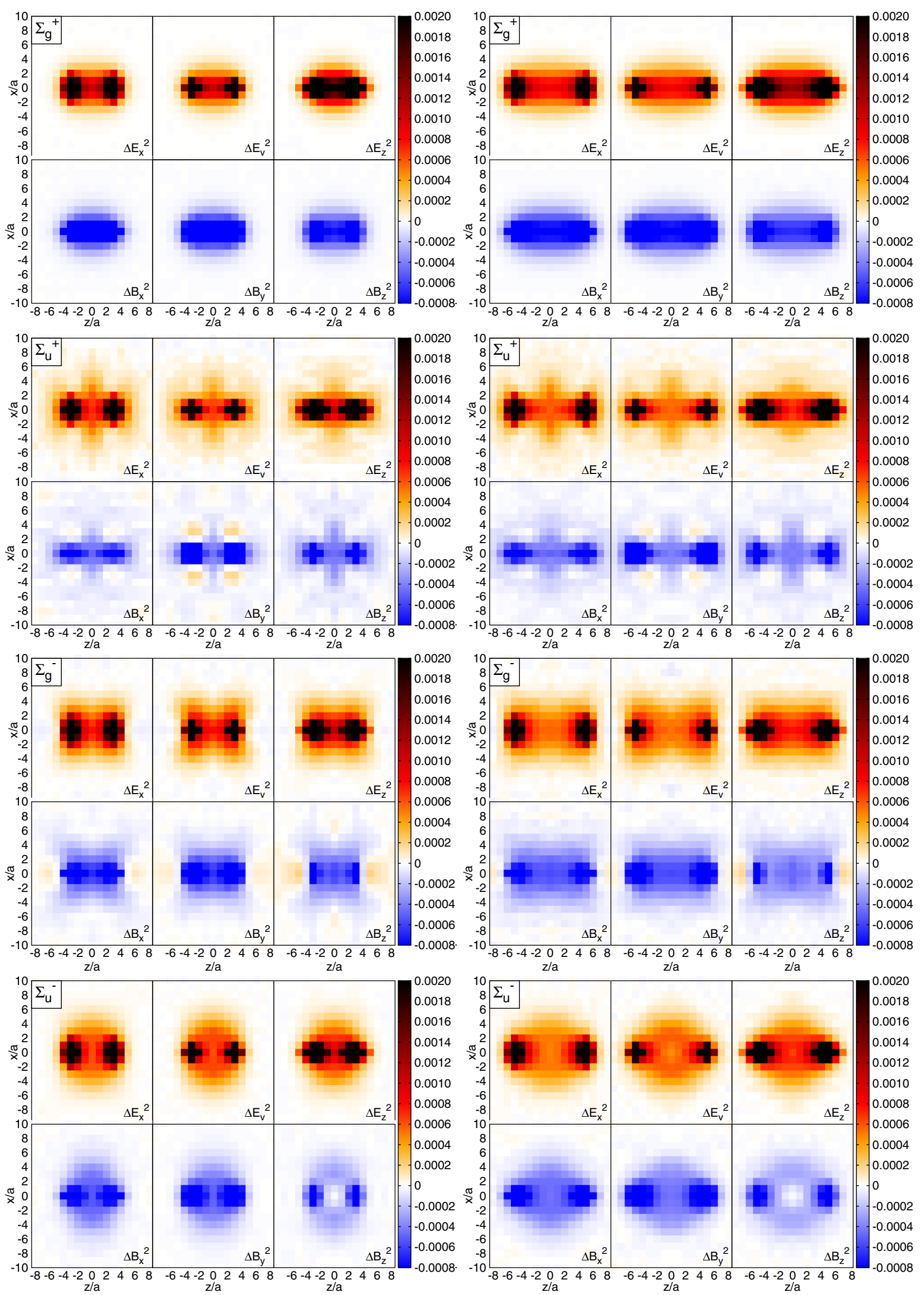

FIG. 9. Flux densities $\Delta F_{j, \Lambda_{\eta}^{c}}^{2}(r ; \mathbf{x}=(x, 0, z)), j=x, y, z$ in the separation plane for gauge group $\mathrm{SU}(2)$ and sectors $\Lambda_{\eta}^{e}=\Sigma_{g}^{+}, \Sigma_{u}^{+}, \Sigma_{g}^{-}, \Sigma_{u}^{-}$. (Left) $Q \bar{Q}$ separation $r=6 a$. (Right) $Q \bar{Q}$ separation $r=10 a$. 

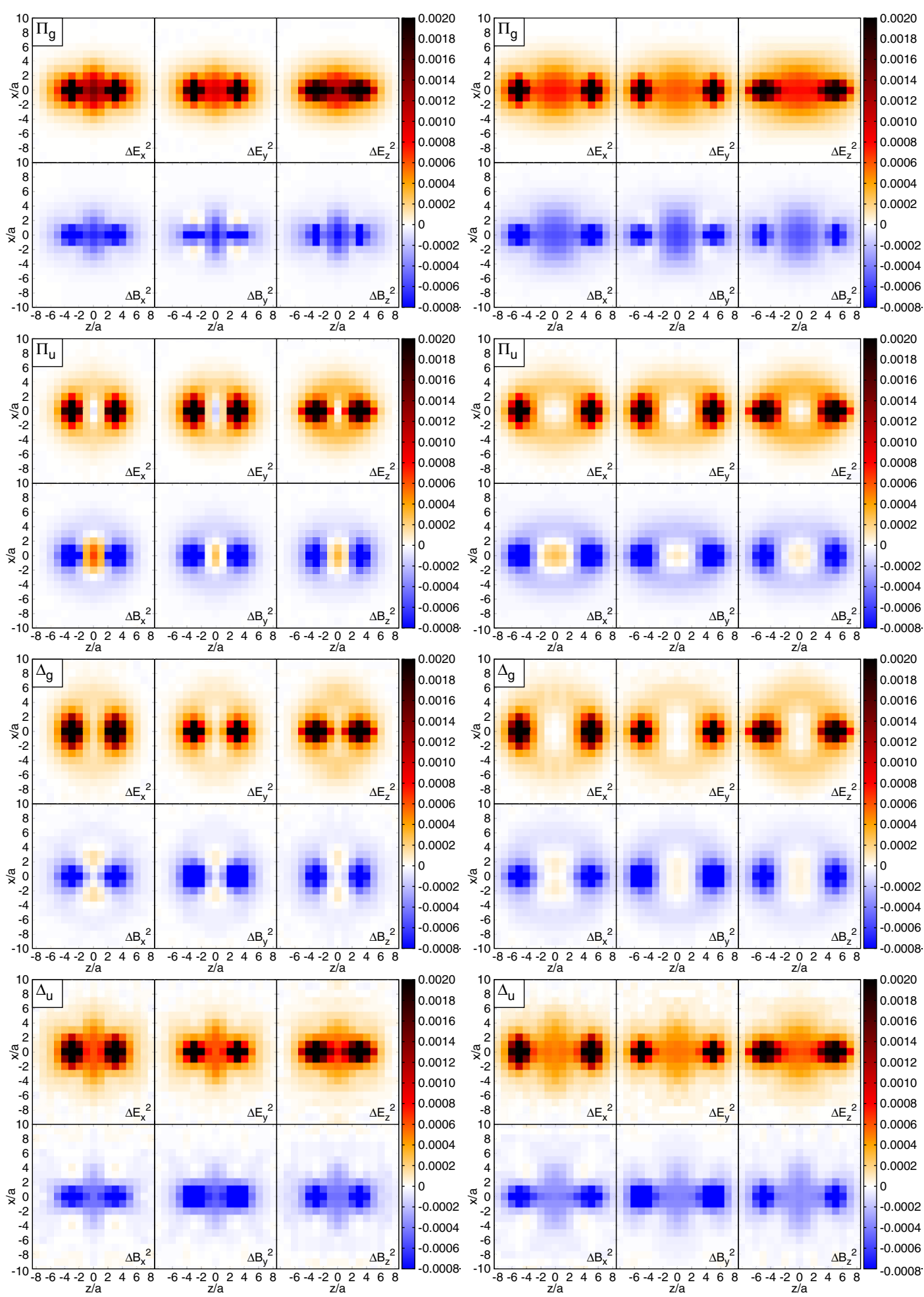

FIG. 10. Flux densities $\Delta F_{j, \Lambda_{\eta}}^{2}(r ; \mathbf{x}=(x, 0, z)), j=x, y, z$ in the separation plane for gauge group $\mathrm{SU}(2)$ and sectors $\Lambda_{\eta}=\Pi_{g}, \Pi_{u}, \Delta_{g}, \Delta_{u}$. (Left) $Q \bar{Q}$ separation $r=6 a$. (Right) $Q \bar{Q}$ separation $r=10 a$.

potentials as local insertions in Wilson loops (see, e.g., Ref. [28]), but numerically, it turned out that they generate less ground state overlap than optimized nonlocal operators (like those discussed in Ref. [31] and in
Sec. II A of this work) and are thus less suited for computations in lattice gauge theory. The leading order gluonic excitation operators of pNRQCD are listed in Table III, where the $Q \bar{Q}$ separation axis is again the $z$ 
TABLE III. Gluonic excitation operators at leading order in the multipole expansion of pNRQCD (the $Q \bar{Q}$ separation axis is the $z$ axis; $D_{j}$ denotes the covariant derivative; see, e.g., Refs. [2,61]).

\begin{tabular}{llll}
\hline \hline$\Sigma_{g}^{+}$ & 1 & $\Sigma_{u}^{+}$ & $D_{z} E_{z}$ \\
$\Sigma_{g}^{-}$ & $D_{z} B_{z}$ & $\Sigma_{u}^{-}$ & $B_{z}$ \\
& & $D_{x} E_{y}-D_{y} E_{x}$ \\
$\Pi_{g}$ & $E_{x}, E_{y}$ & $\Pi_{u}$ & $B_{x}, B_{y}$ \\
& $D_{x} B_{z}-D_{z} B_{x}, D_{y} B_{z}-D_{z} B_{y}$ & & $D_{x} E_{z}-D_{z} E_{x}, D_{y} E_{z}-D_{z} E_{y}$ \\
$\Delta_{g}$ & $D_{x} B_{x}-D_{y} B_{y}$ & $\Delta_{u}$ & $D_{x} E_{x}-D_{y} E_{y}$ \\
& $D_{x} B_{y}+D_{y} B_{x}$ & & $D_{x} E_{y}+D_{y} E_{x}$ \\
\hline \hline
\end{tabular}

axis. For certain $\Lambda_{\eta}^{(\epsilon)}$ sectors, the flux densities we have obtained by our lattice computation closely resemble the pNRQCD operators. For example, in the lower panel of Fig. 8, one can clearly see that the chromomagnetic flux densities for $\Pi_{u}$ and $\Delta_{g}$ are significantly larger than for the ordinary static potential $\Sigma_{g}^{+}$, in particular the $x$ and $y$ components. The corresponding pNRQCD operators include $B_{x}$ and $B_{y}$ as well as $D_{x} B_{x}-D_{y} B_{y}$ and $D_{x} B_{y}+D_{y} B_{x}$. Similarly, for $\Sigma_{u}^{-}$, the $z$ component of the chromomagnetic flux density is rather large, where one of the corresponding pNRQCD operators is $B_{z}$. Further interesting structures are the double peaks in the chromoelectric flux densities for $\Sigma_{u}^{-}$ and $\Pi_{u}$ as shown in the upper panel of Fig. 8. The pNRQCD operators for these sectors contain derivatives in the $x$ direction of the corresponding chromoelectric field operators, $D_{x} E_{y}-D_{y} E_{x}$ and $D_{x} E_{z}-D_{z} E_{x}$, respectively. Again, this is consistent because from lattice gauge theory it is known that such derivative operators generate nodes in the corresponding wave functions.

Finally, we compare and discuss our results in the context of a recent and similar lattice computation of hybrid static potential flux densities [51]. There, the flux densities for two hybrid sectors $\Lambda_{\eta}^{(\epsilon)}=\Sigma_{u}^{+}, \Pi_{u}$ were computed for gauge group SU(3), for $\Lambda_{\eta}=\Pi_{u}$ not only for the ground state but also for the first excitation. We have computed the flux densities for the ground states of the seven hybrid sectors $\Lambda_{\eta}^{(\epsilon)}=\Sigma_{u}^{+}, \Sigma_{g}^{-}, \Sigma_{u}^{-}, \Pi_{g}$, $\Pi_{u}, \Delta_{g}, \Delta_{u}$ for gauge groups $\mathrm{SU}(2)$ as well as $\mathrm{SU}(3)$. Lattice spacings, spacetime volumes, and $Q \bar{Q}$ separations are similar in both works. Compared to Ref. [51], our presentation of results is different in the following aspects:

(i) We show flux densities $\Delta F^{2}(r ; \mathbf{x})$ for $j=x, y$ separately, while in Ref. [51], onlly the average of the $x$ and the $y$ component is shown, i.e., $\Delta F_{\perp, \Lambda_{\eta}^{(e)}}^{2}(r ; \mathbf{x})$ [cf. also Eq. (21)].

(ii) In contrast to Ref. [51], we do not show the flux densities on the $Q \bar{Q}$ separation axis $x=y=0$ as curves because several of the hybrid static potentials have small flux densities on the separation axis but large flux densities on spherical shells rather far away. Since the latter information is lost in such 1D curve plots, we prefer to show 2D color maps including the separation axis [see Figs. 9 and 10 for SU(2) and Figs. 13 and 14 for SU(3)].

Comparing the ground state flux densities for $\Lambda_{\eta}^{(\epsilon)}=$ $\Sigma_{u}^{+}, \Pi_{u}$ and gauge group $\mathrm{SU}(3)$, which were computed both in Ref. [51] (see Figs. 7, 10, 11, and 12 in Ref. [51]) and this work (see Figs. 11 to 14), we find fair agreement. A detailed comparison is, however, difficult because of the different $Q \bar{Q}$ separations considered. Concerning statistical errors, our results are more precise by a factor of up to 5. An obvious reasons for this is the larger number of gauge link configurations we have been using (4500 compared to 1199). Moreover, we have improved our statistical precision by averaging data points, which are related by symmetries, e.g., rotational symmetry as discussed in detail in Secs. IIC and IV B. Such a symmetrization was not done in Ref. [51] as indicated by various plots presented in Ref. [51].

\section{CONCLUSIONS}

We have computed chromoelectric and chromomagnetic flux densities for hybrid static potential states for seven different sectors $\Lambda_{\eta}^{(\epsilon)}=\Sigma_{u}^{+}, \Sigma_{g}^{-}, \Sigma_{u}^{-}, \Pi_{g}, \Pi_{u}, \Delta_{g}, \Delta_{u}$ both in $\mathrm{SU}(2)$ and $\mathrm{SU}(3)$ lattice gauge theory. These flux densities can be interpreted as flux densities inside heavy hybrid mesons and thus provide insights into the structure of such mesons. Five of these sectors, $\Lambda_{\eta}^{(\epsilon)}=\Sigma_{g}^{-}, \Sigma_{u}^{-}, \Pi_{g}, \Delta_{g}, \Delta_{u}$, are investigated for the first time in this work, while our computation of the remaining two sectors, $\Lambda_{\eta}^{(\epsilon)}=\Sigma_{u}^{+}, \Pi_{u}$, confirms results recently published [51], now provided with improved precision. We find flux tubes with interesting structure, significantly different from that of the ordinary static potential with $\Lambda=\Sigma_{g}^{+}$and reminiscent of different vibrational modes of a string. There are also localized peaks in the flux densities, which can be interpreted as valence gluons. Moreover, we compared the resulting flux densities to local operators typically used to study such states, e.g., in pNRQCD. 
Concerning future work, a straightforward direction would be to consider smaller lattice spacings and larger spatial volumes, i.e., to study the continuum and infinite volume limit. However, we do not expect significant changes in the numerical results presented here, since we already have partly investigated discretization errors (by comparing results obtained with unsmeared and with HYP2-smeared static propagators) and finite volume corrections by comparing our $\mathrm{SU}(2)$ main results to an identical computation with a smaller volume and lower statistics]. A more interesting direction would be to extend the computation by including also dynamical light quarks. In principle, one could then study not just heavy-heavy hybrid mesons but, more generally, heavyheavy exotic mesons and explore their gluon and light quark distribution at the same time. In practice, however, this might be very challenging because a hybrid static potential state might decay into the ordinary static potential and one or more light mesons.

\section{ACKNOWLEDGMENTS}

We thank Charlotte Meyerdierks for contributions at an early stage of this work [62]. We acknowledge useful discussions with Pedro Bicudo and Francesca Cuteri. C. R. acknowledges support by a Karin and Carlo Giersch Scholarship of the Giersch foundation. O. P. and M. W. acknowledge support by the German Research Foundation, Grants No. PH 158/4-1 and No. WA 3000/2-1. M. W. acknowledges support by the Heisenberg Programme of the German Research Foundation, Grant No. WA 3000/3-1. This work was supported in part by the Helmholtz International Center for FAIR within the framework of the LOEWE program launched by the State of Hessen. Calculations on the Goethe-HLR and on the on the FUCHS-CSC high-performance computer of the Frankfurt University were conducted for this research. We would like to thank HPC-Hessen, funded by the State Ministry of Higher Education, Research and the Arts, for programming advice.

\section{APPENDIX A: TRANSFORMATION OF $\left|\mathbf{0}_{\Lambda_{\eta}^{\epsilon}}(r)\right\rangle$ UNDER ROTATIONS}

In this Appendix, we derive Eq. (11).

Static potential eigenstates $\left|0_{\lambda_{n}}(r)\right\rangle$ (introduced in Sec. II C for $|\lambda| \geq 1$ ) are also eigenstates of the $z$ component of the total angular momentum operator $J_{z}$ and thus transform under rotations around the $z$ axis with angle $\varphi$ according to

$$
R_{z}(\varphi)\left|0_{\lambda_{\eta}}(r)\right\rangle=e^{i \varphi J_{z}}\left|0_{\lambda_{\eta}}(r)\right\rangle=e^{i \varphi \lambda}\left|0_{\lambda_{\eta}}(r)\right\rangle
$$

Consequently,

$$
\begin{aligned}
R_{z}(\varphi)\left(P_{x}\left|0_{\lambda_{\eta}}(r)\right\rangle\right) & =e^{i \varphi J_{z}} P_{x}\left|0_{\lambda_{\eta}}(r)\right\rangle=P_{x} e^{i(-\varphi) J_{z}}\left|0_{\lambda_{\eta}}(r)\right\rangle \\
& =e^{i(-\varphi) \lambda}\left(P_{x}\left|0_{\lambda_{\eta}}(r)\right\rangle\right)
\end{aligned}
$$

$\left(J_{z} P_{x}=-P_{x} J_{z}\right.$ has been used) and

$$
P_{x}\left|0_{+\lambda_{\eta}}(r)\right\rangle=\left|0_{-\lambda_{\eta}}(r)\right\rangle \text {. }
$$

The last equation allows us to express states $\left|0_{\Lambda_{\eta}^{e}}(r)\right\rangle$ for $\Lambda \geq 1$ in terms of states $\left|0_{\lambda_{\eta}}(r)\right\rangle$,

$$
\left|0_{\Lambda_{\eta}^{ \pm}}(r)\right\rangle=\frac{1}{\sqrt{2}}\left(\left|0_{+\lambda_{\eta}}(r)\right\rangle \pm\left|0_{-\lambda_{\eta}}(0)\right\rangle\right),
$$

where $\lambda=\Lambda$. Using Eq. (A1), one can infer

$$
R_{z}(\varphi)\left|0_{\Lambda_{\eta}^{ \pm}}(r)\right\rangle=\cos (\varphi \Lambda)\left|0_{\Lambda_{\eta}^{ \pm}}(r)\right\rangle+i \sin (\varphi \Lambda)\left|0_{\Lambda_{\eta}^{\mp}}(r)\right\rangle
$$

for $\Lambda \geq 1$, which is Eq. (11). Note that Eq. (A5) also holds for $\Lambda=0$ because it reduces to $R_{z}(\varphi)\left|0_{\Lambda_{\eta}^{ \pm}}(r)\right\rangle=\left|0_{\Lambda_{\eta}^{ \pm}}(r)\right\rangle$, i.e., correctly indicates rotational invariance for states with total angular momentum $\Lambda=0$.

\section{APPENDIX B: HYBRID STATIC POTENTIAL FLUX DENSITIES FOR ALL $\Lambda_{\eta}^{\epsilon}$ SECTORS: PLOTS FOR SU(3) GAUGE THEORY}

Hybrid static potential flux densities for SU(3) gauge theory are shown in Figs. 11-14. Qualitatively, these plots are very similar to the corresponding SU(2) plots in Figs. 7 to 10. For a discussion, see Sec. IV C. 

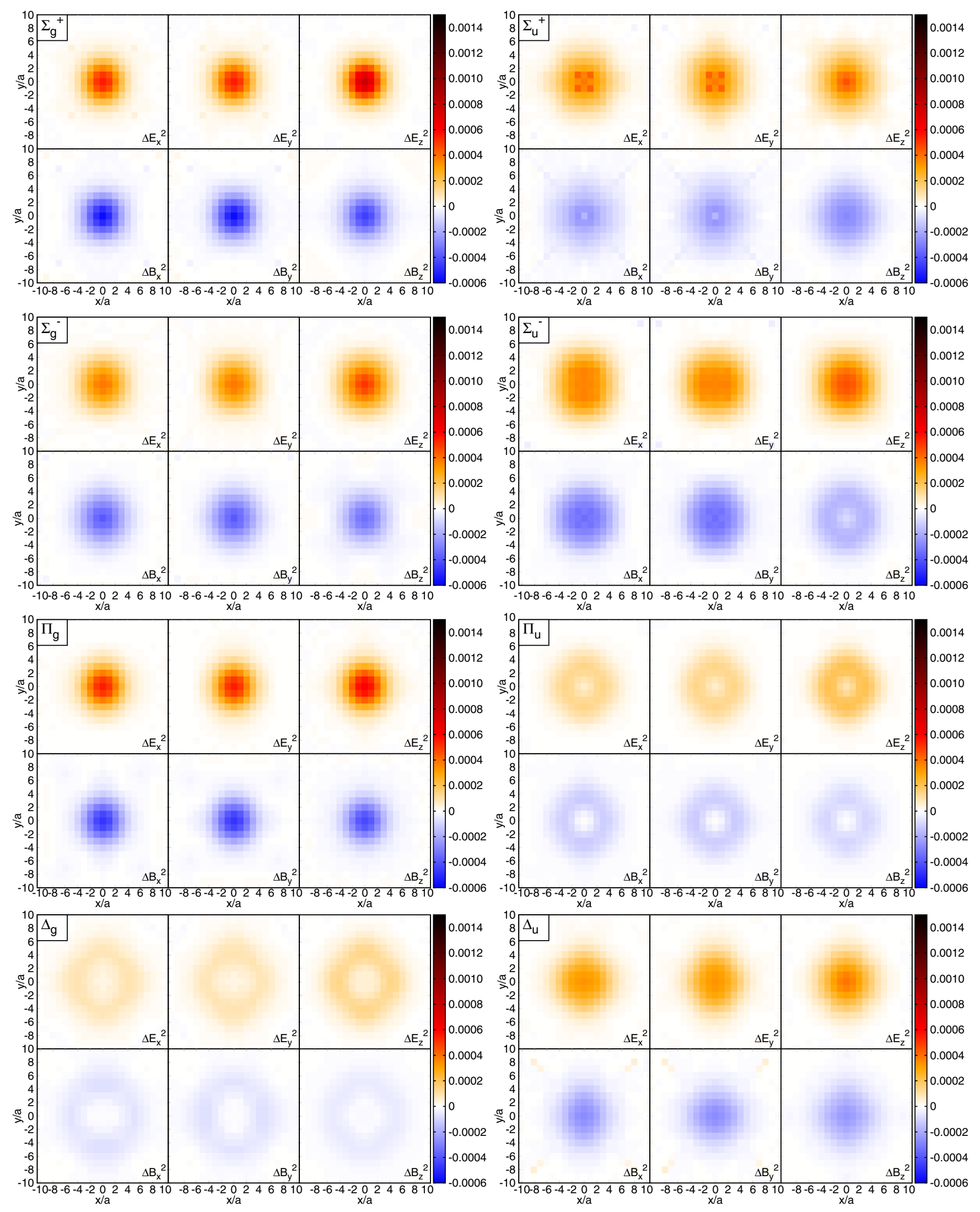

FIG. 11. Flux densities $\Delta F_{j, \Lambda_{j}^{(e)}}^{2}(r ; \mathbf{x}=(x, y, 0)), j=x, y, z$ in the mediator plane for gauge group $\mathrm{SU}(3)$; all investigated sectors $\Lambda_{\eta}^{(\epsilon)}=\Sigma_{g}^{+}, \Sigma_{u}^{+}, \Sigma_{g}^{-}, \Sigma_{u}^{-}, \Pi_{g}, \Pi_{u}, \Delta_{g}, \Delta_{u}$; and $Q \bar{Q}$ separation $r=10 a$. 

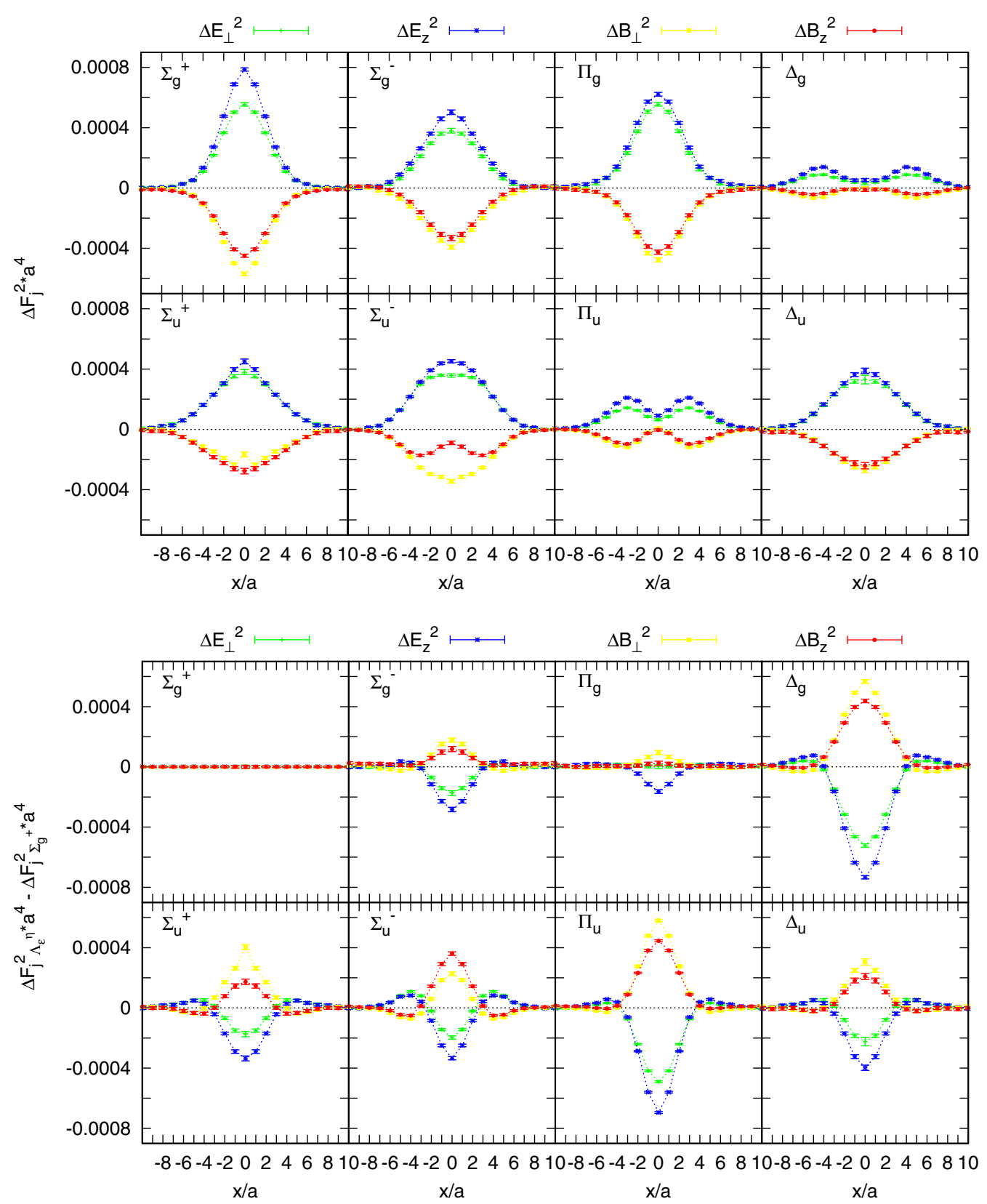

FIG. 12. Flux densities on the $x$ axis for gauge group SU(3); all investigated sectors $\Lambda_{\eta}^{(e)}=\Sigma_{g}^{+}, \Sigma_{u}^{+}, \Sigma_{g}^{-}, \Sigma_{u}^{-}, \Pi_{g}, \Pi_{u}, \Delta_{g}, \Delta_{u}$; and $Q \bar{Q}$ separation $r=10 a$. (Top) $\Delta F_{j, \Lambda_{\eta}^{(e)}}^{2}(r ; \mathbf{x}=(x, 0,0)), j=\perp$, . (Bottom) $\Delta F_{j, \Lambda_{\eta}^{(e)}}^{2}(r ; \mathbf{x}=(x, 0,0))-\Delta F_{j, \Sigma_{g}^{+}}^{2}(r ; \mathbf{x}=(x, 0,0)), j=\perp, z$. 

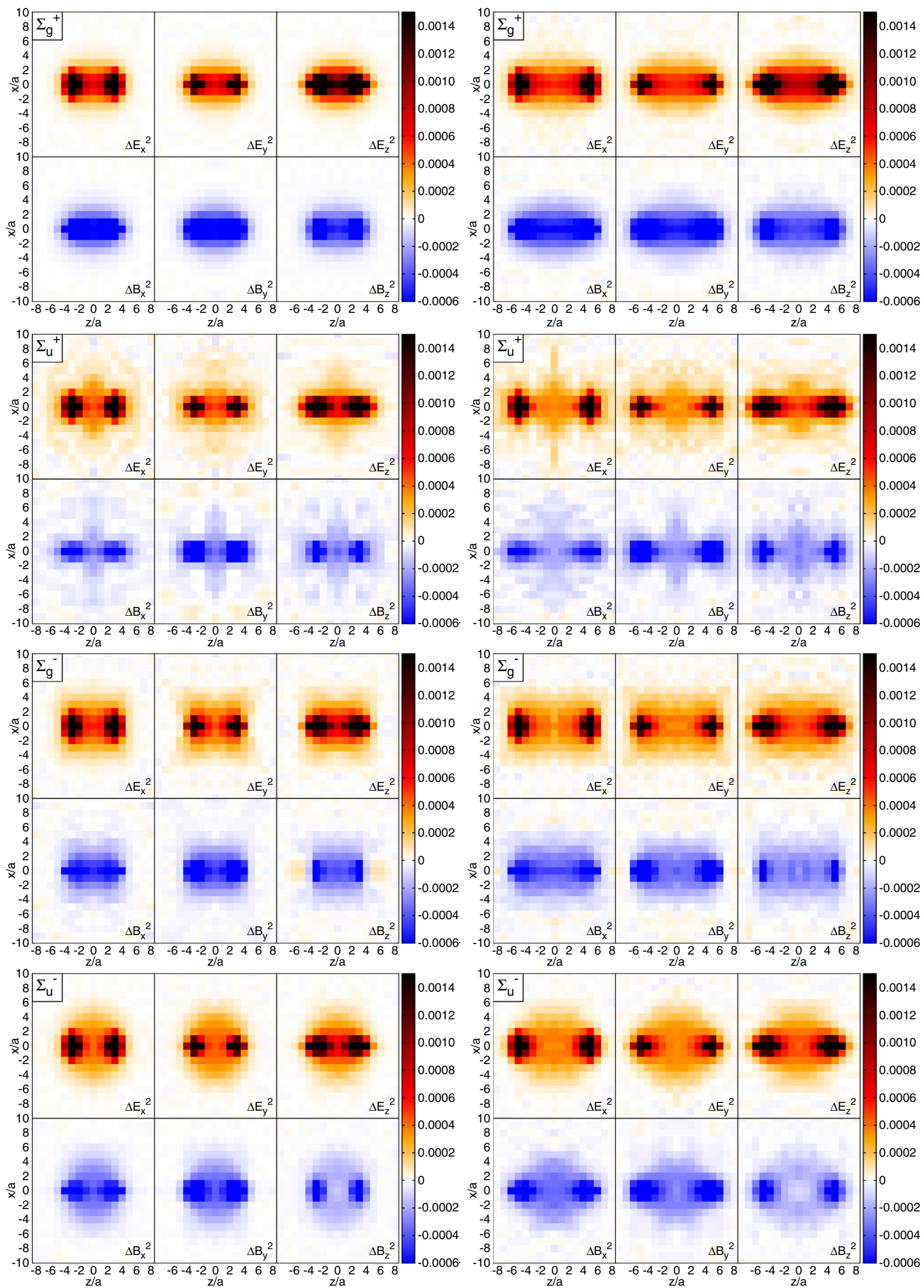

FIG. 13. Flux densities $\Delta F_{j, \Lambda_{\eta}^{\epsilon}}^{2}(r ; \mathbf{x}=(x, 0, z)), j=x, y, z$ in the separation plane for gauge group $\mathrm{SU}(3)$ and sectors $\Lambda_{\eta}^{e}=\Sigma_{g}^{+}, \Sigma_{u}^{+}, \Sigma_{g}^{-}, \Sigma_{u}^{-}$. (Left) $Q \bar{Q}$ separation $r=6 a$. (Right) $Q \bar{Q}$ separation $r=10 a$. 

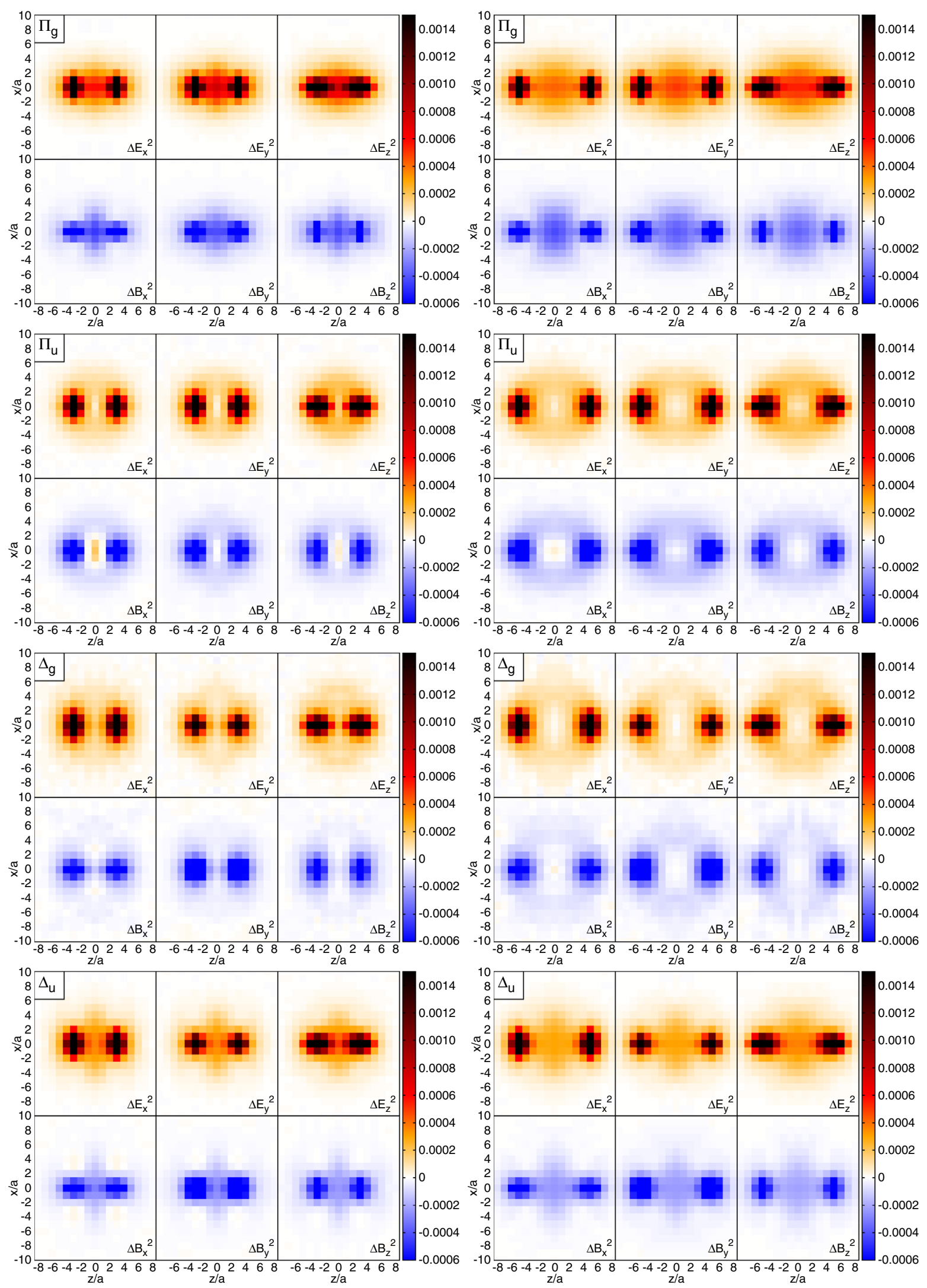

FIG. 14. Flux densities $\Delta F_{j, \Lambda_{\eta}}^{2}(r ; \mathbf{x}=(x, 0, z)), j=x, y, z$ in the separation plane for gauge group $\mathrm{SU}(3)$ and sectors $\Lambda_{\eta}=\Pi_{g}, \Pi_{u}, \Delta_{g}, \Delta_{u}$. (Left) $Q \bar{Q}$ separation $r=6 a$. (Right) $Q \bar{Q}$ separation $r=10 a$. 
[1] S. L. Olsen, T. Skwarnicki, and D. Zieminska, Nonstandard heavy mesons and baryons: Experimental evidence, Rev. Mod. Phys. 90, 015003 (2018).

[2] M. Berwein, N. Brambilla, J. Tarrus Castella, and A. Vairo, Quarkonium hybrids with nonrelativistic effective field theories, Phys. Rev. D 92, 114019 (2015).

[3] E. Braaten, C. Langmack, and D. H. Smith, Selection Rules for Hadronic Transitions of XYZ Mesons, Phys. Rev. Lett. 112, 222001 (2014).

[4] C. A. Meyer and E. S. Swanson, Hybrid mesons, Prog. Part. Nucl. Phys. 82, 21 (2015).

[5] E. S. Swanson, $X Y Z$ states: Theory overview, AIP Conf. Proc. 1735, 020013 (2016).

[6] R. F. Lebed, R. E. Mitchell, and E. S. Swanson, Heavyquark QCD exotica, Prog. Part. Nucl. Phys. 93, 143 (2017).

[7] L. A. Griffiths, C. Michael, and P. E. L. Rakow, Mesons with excited glue, Phys. Lett. 129B, 351 (1983).

[8] N. A. Campbell, L. A. Griffiths, C. Michael, and P. E. L. Rakow, Mesons with excited glue from SU(3) lattice gauge theory, Phys. Lett. 142B, 291 (1984).

[9] N. A. Campbell, A. Huntley, and C. Michael, Heavy quark potentials and hybrid mesons from SU(3) lattice gauge theory, Nucl. Phys. B306, 51 (1988).

[10] C. Michael and S. J. Perantonis, Potentials and glueballs at large beta in SU(2) pure gauge theory, J. Phys. G 18, 1725 (1992).

[11] S. Perantonis and C. Michael, Static potentials and hybrid mesons from pure SU(3) lattice gauge theory, Nucl. Phys. B347, 854 (1990).

[12] K. J. Juge, J. Kuti, and C. J. Morningstar, Gluon excitations of the static quark potential and the hybrid quarkonium spectrum, Nucl. Phys. B, Proc. Suppl. 63, 326 (1998).

[13] M. J. Peardon, Coarse lattice results for glueballs and hybrids, Nucl. Phys. B, Proc. Suppl. 63, 22 (1998).

[14] K. J. Juge, J. Kuti, and C. J. Morningstar, A study of hybrid quarkonium using lattice QCD, AIP Conf. Proc. 432, 136 (1998).

[15] C. Morningstar, K. J. Juge, and J. Kuti, Gluon excitations of the static quark potential, arXiv:hep-lat/9809015.

[16] C. Michael, Hadronic spectroscopy from the lattice: Glueballs and hybrid mesons, Nucl. Phys. A655, c12 (1999).

[17] K. J. Juge, J. Kuti, and C. J. Morningstar, Ab Initio Study of Hybrid $\bar{b} g b$ Mesons, Phys. Rev. Lett. 82, 4400 (1999).

[18] K. J. Juge, J. Kuti, and C. J. Morningstar, The heavy hybrid spectrum from NRQCD and the Born-Oppenheimer approximation, Nucl. Phys. B, Proc. Suppl. 83, 304 (2000).

[19] C. Michael, Quarkonia and hybrids from the lattice, Proc. Sci., HF8 (1999) 001 [arXiv:hep-ph/9911219].

[20] G. S. Bali, B. Bolder, N. Eicker, T. Lippert, B. Orth, P. Ueberholz, K. Schilling, and T. Struckmann (SESAM and $T \chi L$ Collaborations), Static potentials and glueball masses from QCD simulations with Wilson sea quarks, Phys. Rev. D 62, 054503 (2000).

[21] C. Morningstar, Gluonic excitations in lattice QCD: A brief survey, AIP Conf. Proc. 619, 231 (2002).

[22] K. J. Juge, J. Kuti, and C. Morningstar, Fine Structure of the QCD String Spectrum, Phys. Rev. Lett. 90, 161601 (2003).
[23] C. Michael, Exotics, Int. Rev. Nucl. Phys. 9, 103 (2004).

[24] K. J. Juge, J. Kuti, and C. Morningstar, The heavy quark hybrid meson spectrum in lattice QCD, AIP Conf. Proc. 688, 193 (2004).

[25] C. Michael, Hybrid mesons from the lattice, arXiv:hep-ph/ 0308293

[26] G. S. Bali and A. Pineda, QCD phenomenology of static sources and gluonic excitations at short distances, Phys. Rev. D 69, 094001 (2004).

[27] K. J. Juge, J. Kuti, and C. Morningstar, Excitations of the static quark anti-quark system in several gauge theories, arXiv:hep-lat/0312019.

[28] P. Wolf and M. Wagner, Lattice study of hybrid static potentials, J. Phys. Conf. Ser. 599, 012005 (2015).

[29] C. Reisinger, S. Capitani, O. Philipsen, and M. Wagner, Computation of hybrid static potentials in SU(3) lattice gauge theory, EPJ Web Conf. 175, 05012 (2018).

[30] C. Reisinger, S. Capitani, L. Müller, O. Philipsen, and M. Wagner, Computation of hybrid static potentials from optimized trial states in SU(3) lattice gauge theory, Proc. Sci., LATTICE2018 (2018) 054 [arXiv:1810.13284].

[31] S. Capitani, O. Philipsen, C. Reisinger, C. Riehl, and M. Wagner, Precision computation of hybrid static potentials in SU(3) lattice gauge theory, Phys. Rev. D 99, 034502 (2019).

[32] B. B. Brandt and M. Meineri, Effective string description of confining flux tubes, Int. J. Mod. Phys. A 31, 1643001 (2016).

[33] M. Fukugita and T. Niuya, Distribution of chromoelectric flux in SU(2) lattice gauge theory, Phys. Lett. 132B, 374 (1983).

[34] J. W. Flower and S. W. Otto, The field distribution in SU(3) lattice gauge theory, Phys. Lett. 160B, 128 (1985).

[35] J. Wosiek and R. W. Haymaker, On the space structure of confining strings, Phys. Rev. D 36, 3297 (1987).

[36] A. Di Giacomo, M. Maggiore, and S. Olejnik, Evidence for flux tubes from cooled QCD configurations, Phys. Lett. B 236, 199 (1990).

[37] A. Di Giacomo, M. Maggiore, and S. Olejnik, Confinement and chromoelectric flux tubes in lattice QCD, Nucl. Phys. B347, 441 (1990).

[38] P. Cea and L. Cosmai, Lattice investigation of dual superconductor mechanism of confinement, Nucl. Phys. B, Proc. Suppl. 30, 572 (1993).

[39] G. S. Bali, K. Schilling, and C. Schlichter, Observing long color flux tubes in SU(2) lattice gauge theory, Phys. Rev. D 51, 5165 (1995).

[40] P. Skala, M. Faber, and M. Zach, Magnetic monopoles and the dual London equation in SU(3) lattice gauge theory, Nucl. Phys. B494, 293 (1997).

[41] G. S. Bali, C. Schlichter, and K. Schilling, Probing the QCD vacuum with static sources in maximal Abelian projection, Prog. Theor. Phys. Suppl. 131, 645 (1998).

[42] N. Cardoso, M. Cardoso, and P. Bicudo, Inside the SU(3) quark-antiquark QCD flux tube: Screening versus quantum widening, Phys. Rev. D 88, 054504 (2013).

[43] P. Cea, L. Cosmai, F. Cuteri, and A. Papa, Flux tubes in the SU(3) vacuum: London penetration depth and coherence length, Phys. Rev. D 89, 094505 (2014). 
[44] P. Cea, L. Cosmai, F. Cuteri, and A. Papa, Flux tubes at finite temperature, J. High Energy Phys. 06 (2016) 033.

[45] P. Cea, L. Cosmai, F. Cuteri, and A. Papa, Flux tubes in the QCD vacuum, Phys. Rev. D 95, 114511 (2017).

[46] P. Cea, L. Cosmai, F. Cuteri, and A. Papa, QCD flux tubes across the deconfinement phase transition, EPJ Web Conf. 175, 12006 (2018).

[47] M. Baker, P. Cea, V. Chelnokov, L. Cosmai, F. Cuteri, and A. Papa, Isolating the confining color field in the SU(3) flux tube, Eur. Phys. J. C 79, 478 (2019).

[48] M. Baker, P. Cea, V. Chelnokov, L. Cosmai, F. Cuteri, and A. Papa, Spatial structure of the color field in the SU(3) flux tube, Proc. Sci., LATTICE2018 (2018) 253 [arXiv:1811.00081].

[49] P. Bicudo, M. Cardoso, and N. Cardoso, Colour fields of the quark-antiquark excited flux tube, EPJ Web Conf. 175, 14009 (2018).

[50] L. Müller and M. Wagner, Structure of hybrid static potential flux tubes in $S U(2)$ lattice Yang-Mills theory, Acta Phys. Pol. B Proc. Suppl. 11, 551 (2018).

[51] P. Bicudo, N. Cardoso, and M. Cardoso, Color field densities of the quark-antiquark excited flux tubes in SU(3) lattice QCD, Phys. Rev. D 98, 114507 (2018).

[52] L. Müller, O. Philipsen, C. Reisinger, and M. Wagner, Structure of hybrid static potential flux tubes in lattice Yang-Mills theory, Proc. Sci., Confinement13 (2018) 053.

[53] H. J. Rothe, Lattice gauge theories: an Introduction, World Sci. Lect. Notes Phys. 82, 1 (2012).
[54] R. G. Edwards, and B. Joó (SciDAC, LHPC, and UKQCD Collaborations), The chroma software system for lattice QCD, Nucl. Phys. B, Proc. Suppl. 140, 832 (2005).

[55] O. Philipsen and M. Wagner, On the definition and interpretation of a static quark anti-quark potential in the colour-adjoint channel, Phys. Rev. D 89, 014509 (2014).

[56] K. Jansen, C. Michael, A. Shindler, and M. Wagner (ETM Collaboration), The static-light meson spectrum from twisted mass lattice QCD, J. High Energy Phys. 12 (2008) 058.

[57] A. Hasenfratz and F. Knechtli, flavour symmetry and the static potential with hypercubic blocking, Phys. Rev. D 64, 034504 (2001).

[58] M. Della Morte, S. Dürr, J. Heitger, H. Molke, J. Rolf, A. Shindler, and R. Sommer, Lattice HQET with exponentially improved statistical precision, Phys. Lett. B 581, 93 (2004).

[59] M. Della Morte, A. Shindler, and R. Sommer, On lattice actions for static quarks, J. High Energy Phys. 08 (2005) 051.

[60] M. Baker, New constraint on effective field theories of the QCD flux tube, Phys. Rev. D 93, 054012 (2016).

[61] N. Brambilla, A. Pineda, J. Soto, and A. Vairo, Potential NRQCD: An effective theory for heavy quarkonium, Nucl. Phys. B566, 275 (2000).

[62] C. Meyerdierks, Investigation of the structure of static potential flux tubes, B.S. thesis, Goethe-Universität Frankfurt am Main, 2017, https://th.physik.uni-frankfurt .de/ mwagner/theses/BA_Meyerdierks.pdf. 